\title{
Fixation-related brain activity during semantic integration of object-scene information
}

\author{
Moreno I. Coco ${ }^{1,2}$, Antje Nuthmann ${ }^{3}$ and Olaf Dimigen ${ }^{4}$
}

1. School of Psychology, The University of East London, London, UK

2. Faculdade de Psicologia, Universidade de Lisboa, Lisbon, Portugal

3. Department of Psychology, Christian-Albrechts-Universität Kiel, Kiel, Germany

4. Department of Psychology, Humboldt-Universität zu Berlin, Berlin, Germany

This research was supported by the Leverhulme Trust (grant ECF-014-205) and Fundação para a Ciência e Tecnologia (grant PTDC/PSI-ESP/30958/2017) to M. I. C, while he was a Research Fellow at the University of Edinburgh. The authors thank Benedikt Ehinger for helpful exchanges about EEG deconvolution techniques. Please address correspondence to moreno.cocoi@gmail.com or olaf.dimigen@ hu-berlin.de. 


\begin{abstract}
In vision science, a topic of great interest and considerable controversy is the processing of objects that are (in)consistent with the overall meaning of the scene in which they occur. How quickly can we access the semantic properties of objects and does this happen before the object is directly looked at? Here we brought novel evidences to this debate by co-registering eye-movements and EEG while participants freely explored photographs of indoor scenes. Each scene contained a target object that was either consistent or inconsistent with the scene context (e.g., toothpaste vs. flashlight in a bathroom). Eye-movement behaviour showed that inconsistent objects were not only more effortful to process (i.e., looked at longer), but also prioritized over consistent objects while they were still in extrafoveal vision (i.e., looked at earlier after scene onset). In fixation-related brain potentials (FRPs), we used a linear deconvolution technique to compare the activity elicited by semantically consistent versus inconsistent objects at scene onset (i.e. in the stimulus-locked ERP), during the target fixation $(t)$, and during the preceding fixation $(t-1)$. We demonstrate a fixation-related N300/N400 effect, whereby inconsistent objects elicited a larger negative shift of FRP activity than consistent objects, already at the pre-target fixation $t-1$ and throughout fixation $t$. Taken together, our results suggest that object meaning can be, at least partly, extracted in extrafoveal vision, and highlight how attentional and neural mechanisms can be simultaneously investigated to uncover the mechanisms of objectscene integration during natural viewing.
\end{abstract}

Keywords: object-scene integration; foveal and peripheral vision; semantic processing; fixationrelated potentials, eye tracking, N300/N400, regression-ERPs 


\section{Introduction}

In our daily activities, for example when we search for something in a room, our attention is mostly oriented to objects. The time course of object recognition and the role of overt attention in this process are therefore topics of considerable interest in the visual sciences. In the context of real-world scene perception, the question of what constitutes an object is a more complex question than intuition would suggest (e.g., Wolfe, Alvarez, Rosenholtz, Kuzmova, \& Sherman, 2011). An object is likely a hierarchical construct (e.g., Feldman, 2003), with both low-level features (e.g., visual saliency) and high-level properties (e.g., semantics) contributing to its identity. Accordingly, when a natural scene is inspected with eye-movements, the observer's attentional selection is thought to be based either on objects (e.g., Nuthmann \& Henderson, 2010), image features (saliency; Itti, Koch, \& Niebur, 1998) or some combination of the two (e.g., Stoll, Thrun, Nuthmann, \& Einhäuser, 2015).

An early and uncontroversial finding is that the recognition of object is mediated by their semantic consistency. For example, an object that the observer would not expect to occur in a particular scene (e.g., a toothbrush in a kitchen) is recognized less accurately (e.g., Biederman, 1972, Davenport \& Potter, 2004, Fenske, Aminoff, Gronau, \& Bar, 2006) and looked at for longer that an expected object (e.g., Cornelissen \& Võ, 2017; De Graef, Christiaens, \& D’Ydewalle, 1990; Henderson, Weeks Jr, \& Hollingworth, 1999).

What is more controversial, however, is the exact time course along which the meaning of an object is processed and how this semantic processing then influences the overt allocation of visual attention (see Wu, Wick, \& Pomplun, 2014, for a recent review). Two interrelated questions are at the core of this debate: (1) How much time is needed to access the meaning of objects after a scene is displayed and (2) can object semantics be extracted before the object is overtly attended, that is, while the object is still outside of high-acuity foveal vision ( $>1^{\circ}$ eccentricity) or even in the periphery $\left(>5^{\circ}\right.$ eccentricity). Evidence that the meaning of not-yet-fixated objects can capture overt attention comes from experiments that have used sparse displays of several standalone objects (e.g., Belke, Humphreys, Watson, Derrick, Meyer, \& Telling, 2008; Moores, Laiti, \& Chelazzi, 2003). For example, Belke et al. showed that the very first saccade in the display was directed more often to a competitor object that was semantically associated with the target object as compared to semantically unrelated distractors.

Whether such findings also generalize to objects embedded in real-world scenes is currently an open question. In fact, the size of the visual span - the area of the visual field from which observers can take in useful information (see Rayner, 2014 for review) - is large in scene viewing. For objectin-scene search, it corresponds to approximately $8^{\circ}$ in each direction from fixation (Nuthmann, 2013). Objects that are highly salient (i.e., visually distinct) are preferentially selected for fixation (e.g., Stoll et al., 2015). If high-level semantic processing also takes place outside the fovea, then objects that are inconsistent with the scene context (which are also thought to be more informative, Antes, 1974) 
should also be fixated earlier in time than consistent ones (Loftus \& Mackworth, 1978; Mackworth \& Morandi, 1967).

However, results from eye-movement studies on this issue have been mixed. A number of studies have indeed reported evidence for an inconsistent object advantage (e.g., Bonitz \& Gordon, 2008; LaPointe \& Milliken, 2016; Loftus \& Mackworth, 1978; Underwood, Templeman, Lamming, \& Foulsham, 2008). Among these studies, only Loftus \& Mackworth, 1978 have reported evidence for an immediate extrafoveal attentional capture (i.e. within the first fixation) by object-scene semantics. In this study, which used relatively sparse line drawings of scenes, the mean amplitude of the saccade into the critical object was more than $7^{\circ}$, suggesting that viewers could process semantic information based on peripheral information obtained in a single fixation. Several other studies, however, have failed to find any advantage for inconsistent objects in attracting overt attention (e.g., De Graef, Christiaens, \& D’Ydewalle, 1990; Henderson, Weeks, \& Hollingworth, 1999; Võ \& Henderson, 2009, 2011). In these experiments, only measures of foveal processing - such as gaze duration - were influenced by object-scene consistency, with longer fixations times on inconsistent than on consistent objects.

The processing of object-scene inconsistencies and its time course have also been investigated in electrophysiological studies (e.g., Ganis \& Kutas, 2003; Mudrik, Lamy, \& Deouell, 2010). In eventrelated potentials (ERPs), it is commonly found that scene-inconsistent objects elicit a larger negative brain response compared to consistent ones. This long-lasting negative shift typically starts as early as 200-250 ms after stimulus onset (Mudrik, Shalgi, Lamy, \& Deouell, 2014; Draschkow, Heikel, Võ, Fiebach, \& Sassenhagen, 2018) and has its maximum at frontocentral scalp sites, in contrast to the centroparietal N400 effect for words (e.g., Kutas \& Federmeier, 2011). The effect was found when the object appeared at a cued location after the scene background was already shown (Ganis \& Kutas, 2003), for objects that were photoshopped into the scene (Mudrik, Lamy, \& Deouell, 2010; Mudrik, et al., 2014; Coco, Araujo, \& Petersson, 2017), and for objects that were part of realistic photographs (Võ \& Wolfe, 2013). ERP effects of object-scene consistency have typically been subdivided into two distinct components: N300 and N400. The earlier part of the negative response, usually referred to as $\mathrm{N} 300$, has been taken to reflect the context-dependent difficulty of object identification, whereas the later N400 has been linked to semantic integration processes after the object is identified (e.g., Dyck $\&$ Brodeur, 2015). The present study was not designed to differentiate between these two subcomponents, especially considering that their scalp distribution is strongly overlapping or even topographically indistinguishable (Draschkow et al., 2018). Thus, for reasons of simplicity, we will in most cases simply refer to all frontocentral negativities as "N400" in the current study, but this term is meant to include the earlier N300 part of the effect.

One limiting factor of these existing ERP studies is that the data were gathered using steadyfixation paradigms in which the free exploration of the scene through eye-movements was not 
permitted. Instead, the critical object was typically large and/or located relatively close to the centre of the screen, and ERPs were time-locked to the onset of the image (e.g., Mudrik, Lamy, \& Deouell, 2010). Due to these limitations, it remains unclear whether foveation of the object is a necessary condition for processing object-scene consistencies, or whether such processing can at least begin in extrafoveal vision.

In the current study, we used fixation-related potentials (FRPs), EEG waveforms aligned to fixation onset, to shed new light on the controversial findings of the role of foveal versus extrafoveal vision in extracting object semantics, while providing insights into the patterns of brain activity that underlie them (for reviews about FRPs see Dimigen, Sommer, Hohlfeld, Jacobs, \& Kliegl, 2011; Nikolaev, Meghanathan, \& van Leeuwen, 2016). FRPs have initially been used to investigate the brain-electric correlates of natural reading, as opposed to serial word presentation, helping researchers to provide finer details about the online processing of linguistic features (such as word predictability, Kretzschmar, Bornkessel-Schlesewsky, \& Schlesewsky, 2009; Dimigen, et al., 2011; Kliegl, Dambacher, Dimigen, Jacobs, \& Sommer, 2012) or the dynamics of the perceptual span during reading (e.g., parafovea-on-fovea effects, Niefind \& Dimigen, 2016). More recently, the coregistration method has also been applied to investigate other naturalistic viewing situations (e.g., Devillez, Guyader, \& Guerin-Dugue, 2015; Kamienkowski, Ison, Quiroga, \& Sigman, 2012; Kaunitz et al., 2014; Rämä \& Baccino, 2010). Much of this research has focussed on the large P300 component elicited by the fixation of task-relevant target stimuli during visual search.

In the present study, we simultaneously recorded eye-movements and FRPs during the viewing of real-world scenes to distinguish between three alternative hypotheses on object-scene integration that can be derived from the literature: (A) one glance of the scene is sufficient to extract object semantics from extrafoveal vision (e.g., Loftus \& Mackworth, 1978), (B) extrafoveal processing of object-scene semantics is possible but takes some time to unfold (e.g., Bonitz \& Gordon, 2008; Underwood et al., 2008), and (C) the processing of object semantics requires foveal vision, that is, a direct fixation of the critical object (e.g., De Graef et al., 1990; Henderson et al., 1999; Võ \& Henderson, 2009).

For the behavioural data, these hypotheses translate as follows: under (A), the probability of immediate target fixation should reveal that already the first saccade on the scene goes more often towards inconsistent than consistent objects. Under (B), there should be no effect on the first eyemovement, but the latency to first fixation on the critical object should still be shorter for inconsistent than consistent objects. Under (C), only fixation times on the critical object itself should differ as a function of object-scene consistency, with longer gaze durations on inconsistent objects.

For the electrophysiological data analysis, we used a novel regression-based analysis approach (linear deconvolution modelling; Dandekar, Privitera, Carney, \& Klein, 2011; Ehinger \& Dimigen, 2018; Smith \& Kutas, 2015b), which allowed us to control for the confounding influences of 
overlapping potentials and oculomotor covariates during natural viewing, which can otherwise distort the neural responses (Dimigen et al., 2011; Nikolaev et al., 2016). In the EEG, hypothesis (A) can be tested by computing the ERP time-locked to the onset of the scene on the display, following the traditional approach. Given that the critical objects in our study were not placed directly in the screen centre, which is where observers began viewing the scene, any effect of object-scene congruency in this ERP would suggest that object semantics is rapidly processed in extrafoveal vision, even before the first eye-movement is generated, in line with Loftus \& Mackworth (1978). Under hypothesis (B) we would not expect to see an effect in the scene-onset ERP. Instead, we should find a negative brain potential (N400) for inconsistent as compared to consistent objects in the FRP aligned to the pre-target fixation that precedes the one that first lands on the critical object. Finally, if (C) is correct, an N400 for inconsistent objects should only arise once the critical object is foveated, i.e., in the FRP aligned to the target fixations. In contrast, no consistency effects should appear in the scene-onset ERP or in the FRP aligned to the pre-target fixation.

Figure 1
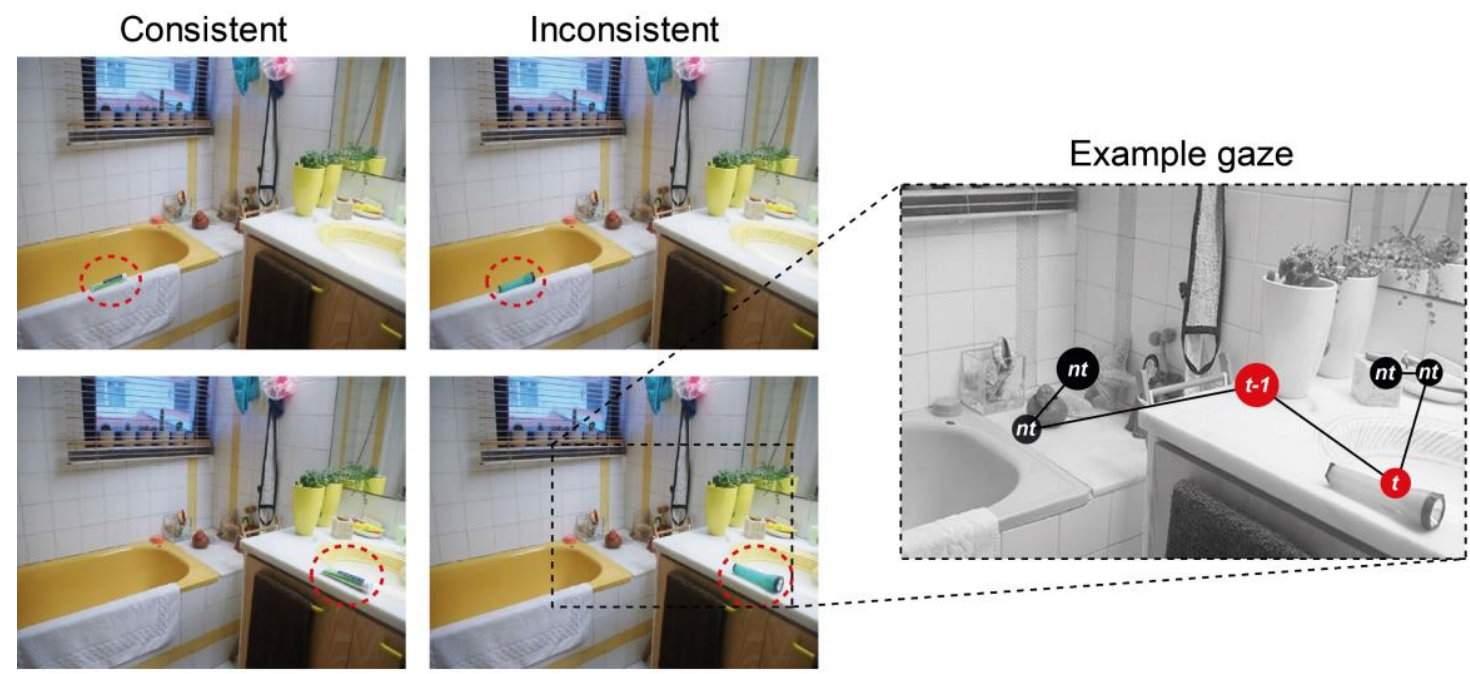

Target object

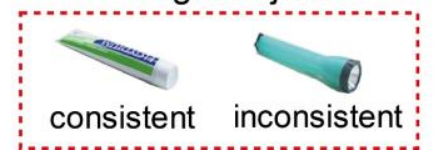

Figure 1. Example stimuli and conditions in the study. Participants viewed photographs of indoor scenes that contained a target object (highlighted with a red circle) that was either semantically consistent (here: toothpaste) or semantically inconsistent (here: flashlight) with the context of the scene. The target object could be placed at different locations within the scene, either on the left or the right side. The example gaze path plotted on the right illustrates the three type of fixations analysed in the study: (a) $t-1$; the fixation preceding the first fixation to the target object, (b) $t$; the first fixation to the target and (c) $n t$; all other (non-target) fixations. Fixation duration is proportional to the diameter of the circle, which is red for the critical fixations, and black for the non-target fixations. 


\section{Methods}

\section{Design and task overview}

We designed a short-term visual working memory change detection task, illustrated in Figure 1 and 2. During the study phase, participants were exposed to photographs of indoor scenes (e.g., a bathroom), each of which contained a target object that was either semantically consistent (e.g., toothpaste) or inconsistent (e.g., a flashlight) with the scene context. In the following recognition phase, after a short retention interval of $900 \mathrm{~ms}$, the same scene was shown again, but in half of the trials either the identity, the location, or both the identity and location of the target object had changed relative to the study phase.

The participants' task was to indicate with a keyboard press whether or not a change had happened to the scene (see LaPointe \& Milliken, 2016, for related research using a change detection task). All eye-movement and EEG analyses in the present paper focus on the semantic consistency manipulation of the target object during the study phase.

\section{Participants}

Twenty-four participants ( 9 male) between the ages of 18 and 33 (mean: 25.0 years) took part in the experiment after providing written informed consent. They were compensated with $£ 7$ per hour. All participants had normal or corrected-to-normal vision. Data from an additional two participants was recorded but removed from the analysis due to excessive scalp muscle (EMG) activity or skin potentials in the raw EEG. Ethics approval was obtained from the Psychology Research Ethics Committee of the University of Edinburgh.

\section{Apparatus and Recording}

Scenes were presented on a 19" CRT monitor (Iiyama Vision Master Pro 454) at a vertical refresh rate of $75 \mathrm{~Hz}$. At the viewing distance of $60 \mathrm{~cm}$, each scene subtended $35.8^{\circ} \times 26.9^{\circ}$ of visual angle. Eye-movements were recorded monocularly from the dominant eye using an SR Research EyeLink 1000 desktop-mounted system at a sampling rate of $1000 \mathrm{~Hz}$. Eye dominance for each participant was determined with a parallax test. A chin and forehead rest was used to stabilize the participant's head. Nine-point calibrations were run at the beginning of each session and whenever the participant's fixation deviated by $>0.5^{\circ}$ horizontally or $>1^{\circ}$ vertically from a drift correction point presented at trial onset.

The EEG was recorded from 64 active electrodes at a sampling rate of $512 \mathrm{~Hz}$ using BioSemi ActiveTwo amplifiers. Four electrodes, located near the left and right canthus and above and below the right eye, recorded the electro-oculogram (EOG). All channels were referenced against the BioSemi common mode sense (CMS; active electrode) and grounded to a passive electrode. The BioSemi hardware is DC coupled and applies digital low-pass filtering through the A/D-converter's 
decimation filter, which has a 5 th order sync response with a $-3 \mathrm{~dB}$ point at $1 / 5$ th of the sample rate (corresponding approximately to a $100 \mathrm{~Hz}$ low-pass filter).

Offline, the EEG was re-referenced to the average of all scalp electrodes and filtered between 0.2 and $30 \mathrm{~Hz}$ using EEGLAB's (Delorme \& Makeig, 2004) finite impulse response filter (function pop_eegfiltnew.m) with default settings. Eye tracking and EEG were synchronized using shared triggers sent via the parallel port of the stimulus presentation PC to the two recording computers. Synchronization was performed offline using the EYE-EEG extension (v0.8) for EEGLAB (Dimigen et al., 2011). All datasets were aligned with a mean synchronization error $\leq 2 \mathrm{~ms}$ as computed based on trigger alignment after synchronization.

\section{Materials \& Rating}

Stimuli consisted of 192 colour photographs of indoor scenes (e.g., bedrooms, bathrooms, offices). An example is shown in Figure 1. Each scene contained a critical object that was either consistent or inconsistent with the scene context. The consistency of the object was assessed in a pretest rating study by eight naïve participants who were not involved in any other aspect of the study. Each participant rated a subset of the photographs only, because object consistency and location (left vs. right) were counterbalanced across four lists. Together with each scene, raters saw a box with a cropped image of the critical object. They were asked (a) to write down the name for the displayed object, and (b) to respond to the question "How likely is it that this object would be found in this room?" using a six-point Likert scale (1-6). For the object naming, a mean naming agreement of $96.35 \%$ was obtained. Furthermore, consistent objects were judged as significantly more likely (5.78 $\pm 0.57)$ to appear in the scene than inconsistent objects $(1.88 \pm 1.11)$, as confirmed by an independentsamples Kruskal-Wallis $H$-test $\left(\chi^{2}(1)=616.09, p<.001\right)$.

Moreover, we ensured that there was no difference between consistent and inconsistent objects on three important low-level variables: object size (pixels square), distance from the centre of the scene (degrees of visual angle) and mean visual saliency of the object computed using the Adaptive Whitening Saliency model (Garcia-Diaz, Fdez-Vidal, Pardo, \& Dosil, 2012). Paired $t$-tests showed no significant difference between consistency conditions in object size, $t(476)=-1.2, p=0.2$; visual saliency, $t(476)=0.1, p=0.9$; and distance from the centre, $t(476)=0.48, p=0.6$. The average distance of the object centroid from the centre of the scene was $11.96^{\circ}$ and $11.83^{\circ}$ for consistent and inconsistent objects, respectively. 
Figure 2

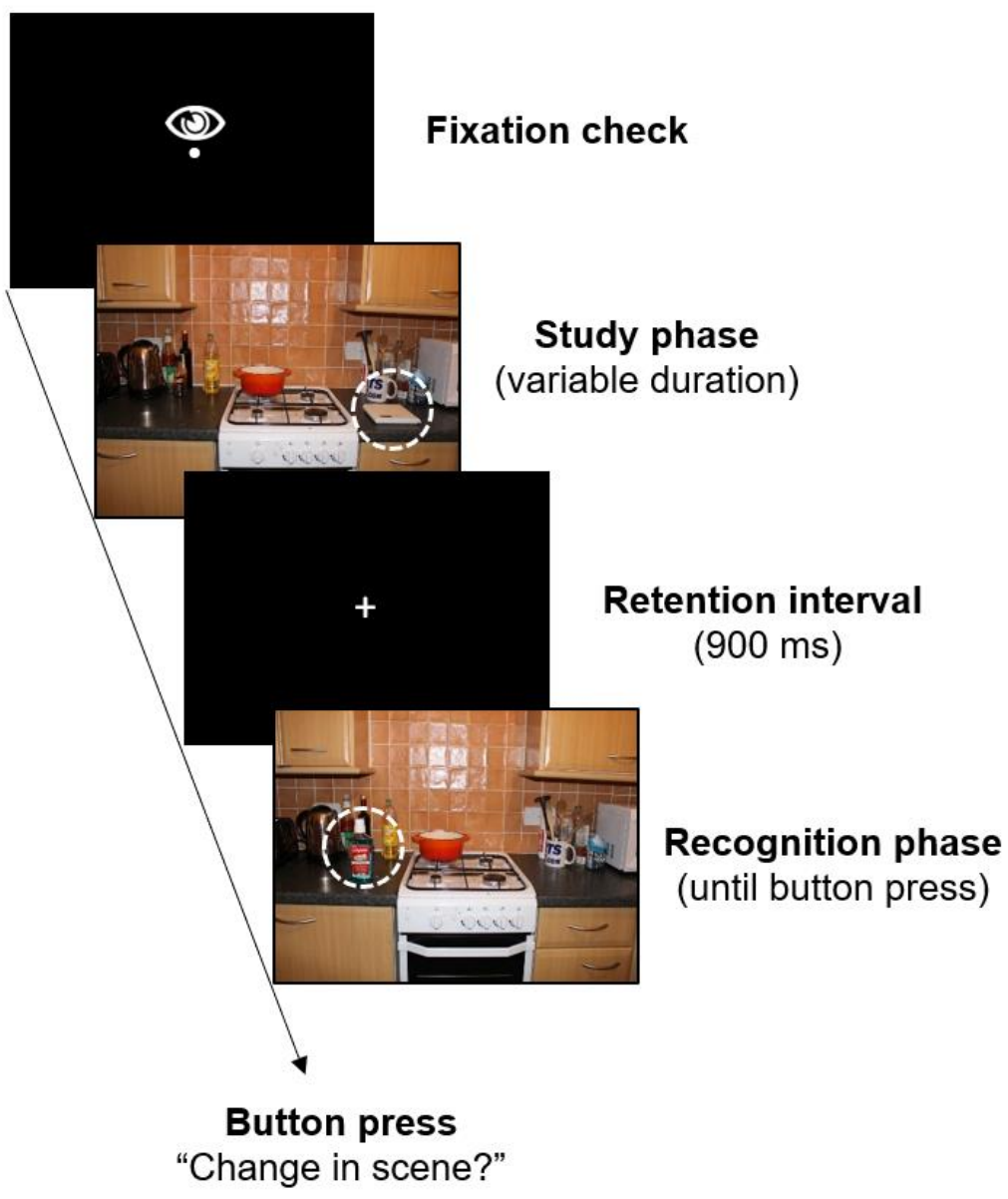

Figure 2. Trial scheme. Following a drift correction, the study scene appeared. The display duration of the scene was controlled by a gaze-contingent mechanism and it disappeared on average $2000 \mathrm{~ms}$ after the target object was fixated. In the following retention interval, only a fixation cross was presented. During the recognition phase, the scene was presented again until participants pressed a button to indicate whether or not a change had occurred within the scene. All analyses in the present paper focus on eye-movement and EEG data collected during the study phase.

\section{Procedure}

A schematic representation of the task is shown in Figure 2. Each trial started with a drift correction of the eye-tracker. Afterwards, the study scene was presented (e.g., a bathroom). The display duration of the study scene was controlled by a gaze-contingent mechanism that ensured that participants fixated the target object (e.g., a toothbrush or flashlight) at least once during the trial. Specifically, the study scene disappeared on average $2000 \mathrm{~ms}$ (with a random jitter of $\pm 200 \mathrm{~ms}$, drawn from a uniform distribution) after the participant had fixated the target object for a minimum duration of $150 \mathrm{~ms}$. The jittered delay of about $2000 \mathrm{~ms}$ was implemented to prevent participants from learning to associate the last fixated object during the study phase with the changed object during the 
recognition phase. If the participant did not fixate the target object within $10 \mathrm{~s}$, the study scene disappeared from the screen and the retention interval was triggered, which lasted $900 \mathrm{~ms}$.

In the following recognition phase (data not analysed here), the scene was presented again, either with (50\% of trials) or without (50\% of trials) a change to an object in the scene. Three types of object changes occurred with equal probability: Location, Consistency, or Both. In the (a) Location condition, the target object changed its position and moved either from left to right or from right to left to another plausible location within the scene (e.g., a toothbrush was placed elsewhere within the scene). In the (b) Consistency condition, the object remained in the same location, but was replaced with another object of opposite semantic consistency (e.g., the toothbrush was replaced by a flashlight or vice-versa). Finally, in the (c) Both condition, the object was both replaced and moved within the scene (e.g., toothbrush was replaced by a flashlight at a different location).

During the recognition phase, participants had to indicate whether they noticed any kind of change within the scene by pressing the arrow keys on the keyboard. Afterwards, the scene disappeared and the next trial began. If participants did not respond within $10 \mathrm{~s}$, a missing response was recorded.

The type of change between trials was fully counterbalanced using a Latin Square rotation. Specifically, the 96 change trials were distributed across 12 different lists, implementing the different types of change. This implies that each participant was exposed to an equal number of consistent and inconsistent change trials. The 96 no-change trials also comprised an equal number of consistent and inconsistent scenes and were the same for each participant. All 192 trials were presented in a randomized order. These trials were preceded by four practice trials at the start of the session. Written instructions were given to explain the task which took 20-40 minutes to complete. The experiment was implemented using the SR Research Experiment Builder software.

\section{Data preprocessing}

\section{Eye-movement events and data exclusion}

Fixations and saccades events were extracted from the raw gaze data using the SR Research Data Viewer software, which performs saccade detection based on velocity and acceleration thresholds of $30^{\circ} \mathrm{s}^{-1}$ and $9,500^{\circ} \mathrm{s}^{-2}$, respectively. In order to provide directly comparable results for eye-movement behaviour and FRP analyses, we discarded all trials on which we did not have clean data from both recordings. Specifically, from a total of 4,608 trials ( 24 participants $\times 192$ trials), we excluded 10 trials $(0.2 \%)$ because of machine error (i.e., data for those trials was not recorded), 689 trials $(15.0 \%)$ because the participant responded incorrectly after the recognition phase and 494 trials $(10.7 \%)$ because the target object was not fixated during the study phase. Finally, we removed an additional 97 trials $(2.1 \%)$ for which the target fixation overlapped with intervals of the EEG that contained non- 
ocular artefacts (see below). The final dataset therefore comprised 3,318 unique trials: 1,631 for the consistent and 1,687 for the inconsistent condition.

\section{EEG ocular artefact correction}

EEG recordings during free viewing are contaminated by three types of ocular artefacts (e.g., Dimigen, 2018; Plöchl, Ossandón, \& König, 2012) which need to be removed to get at the genuine brain activity. Here we applied an optimized variant (Dimigen, 2018) of Independent Component Analysis (ICA, Jung et al., 1998), which uses the information provided by the eye-tracker to objectively identify ocular ICA components (Plöchl et al. (2012).

In a first step, we created optimized ICA training data by high-pass filtering a copy of the EEG at 2 Hz (Winkler, Debener, Müller, \& Tangermann, 2015, Dimigen, 2018) and segmenting it into epochs lasting from scene onset until $3 \mathrm{~s}$ thereafter. This high pass-filtered training data was entered into an extended Infomax ICA using EEGLAB and the resulting unmixing weights were then transferred to the original (i.e. less strictly filtered) recording (Viola, Debener, Thorne, \& Schneider, 2010). From this original EEG dataset, we then removed all independent components whose time course varied more strongly during saccade intervals (defined as lasting from -20 ms before saccade onset until 20 ms after saccade offset) than during fixations with the threshold for the variance ratio (saccade/fixation, see Plöchl et al. 2012) set to 1.3. The artefact-corrected continuous EEG was then back-projected to the sensor space. For a validation of the ICA procedure, please refer to Figure A1 and Dimigen (2018).

In a next step, intervals with residual non-ocular artefacts (e.g., EMG bursts) were detected by shifting a $2000 \mathrm{~ms}$ moving window in steps of $100 \mathrm{~ms}$ across the continuous recording. Whenever the voltages within the window exceeded a peak-to-peak threshold of $120 \mu \mathrm{V}$ in at least one of the channels, all data within the window was marked as "bad" and subsequently excluded from the analysis. Within the linear deconvolution framework (see below), this can easily be done by setting all predictors to zero during these bad EEG intervals (Ehinger \& Dimigen, 2018; Smith \& Kutas, 2015b), meaning that the data in these intervals will not affect the computation.

\section{Analysis}

\section{Eye-movement data}

Dependent measures: Behavioral analyses focused on four eye-movement measures commonly reported in the semantic consistency literature: (a) cumulative probability of having fixated the target 
object as a function of the ordinal fixation number, (b) the probability of immediate object fixation ${ }^{1}$, (c) the latency to first fixation on the target object, and (d) the gaze duration on the target object.

Linear-mixed effect modelling: Eye-movement data were analysed using linear mixed-effects models (LMM) and generalized linear mixed-effects models (GLMM) as implemented in the lme 4 package in R (Bates, Mächler, Bolker, \& Walker, 2015). The only exception was the cumulative probability of first-fixations on the target for which a generalized linear model (GLM) was used. One advantage of (G)LMM modelling is that it allows one to simultaneously model the intrinsic variability of both participants and scenes (e.g., Nuthmann \& Einhäuser, 2015).

In all analyses, the main predictor was the Consistency of the critical object (Consistent $=-0.5$, Inconsistent $=0.5)$ in the study scene. In the (G)LMMs, Participant (24) and Scene (192) were included as random intercepts ${ }^{2}$. The cumulative probability of object fixation was analysed using a GLM with a binomial (probit) link. This model included the Ordinal Number of Fixation on the scene as a predictor; it was entered as a continuous variable ranging from 1 to a maximum of 28 (the $99^{\text {th }}$ quantile).

In the tables of results, we report the beta coefficients, $t$-values (LMM), $z$-values (GLMM), and $p$ values for each model. Level of significance of the coefficients associated with the predictors retained in the model was calculated from an $F$-test based on Satterthwaite approximation to the effective degrees of freedom (Satterthwaite, 1946).

\section{Electrophysiological data}

Linear Deconvolution Modelling (first level of analysis): EEG measurements during active vision are associated with two major methodological problems: overlapping potentials and low-level signal variability. Overlapping potentials arise from the rapid pace of active information sampling through eye-movements, which causes the neural responses that are evoked by subsequent fixations on the stimulus to overlap with each other. Because the average fixation duration usually varies between conditions, this changing overlap can easily confound the measured waveforms. A related issue is the mutual overlap between the ERP elicited by the initial presentation of the stimulus and the FRPs evoked by the subsequent fixations on it (see Dimigen et al., 2011). This second type of overlap is

\footnotetext{
${ }^{1}$ The first central fixation was removed for each trial. Therefore, "first fixation" refers here to the fixation that follows the initial saccade after scene onset.

${ }^{2}$ We did not include random slopes for two reasons: For Participant, the inclusion of a random slope led to a small variance and perfect correlation between intercept and slope. For the predictor Scene, only the change trials were fully counterbalanced in terms of location and consistency, meaning that the slope for Consistency could not be estimated for the no-change trials.
} 
especially important in experiments like ours, in which the critical fixations occurred at different latencies after stimulus onset in the two experimental conditions.

The problem of signal variability refers to the fact that low-level visual and oculomotor variables can also influence the morphology of the predominantly visually-evoked fixation-related neural responses (e.g., Dimigen et al., 2011; Kristensen, Rivet, \& Guerin-Dugué, 2017; Nikolaev et al., 2016). The most relevant of these variables, which is known to modulate the entire FRP waveshape, is the amplitude of the saccade that precedes the onset of the current fixation (e.g., Dandekar et al., 2011; Thickbroom, Knezevič, Carroll, \& Mastaglia, 1991). One option for controlling the effect of saccade amplitude is to include it as a continuous covariate in a massive univariate regression-model (Smith \& Kutas, 2015a, 2015b), in which a separate regression model is computed for each EEG time point and channel (e.g., Weiss et al., 2016). However, this method does not account for overlapping potentials.

A better approach that allows us to simultaneously control for overlapping potentials and lowlevel covariates is deconvolution within the linear model (for tutorial reviews see Smith \& Kutas, 2015a, 2015b; Ehinger \& Dimigen, 2018), sometimes also called continuous-time regression (Smith $\&$ Kutas, 2015b). Initially developed to separate overlapping BOLD responses (Serences, 2004), linear deconvolution has recently also been applied to separate overlapping potentials in ERP (Smith \& Kutas, 2015b) and FRP paradigms (Dandekar et al., 2011; Ehinger \& Dimigen, 2018; Kristensen, Guerin-Dugué, \& Rivet, 2017). Another elegant property of this approach is that ERPs elicited by scene onset and the FRPs elicited by fixations on the scene can be disentangled and simultaneously estimated in the same regression model. The benefits of deconvolution are illustrated in more detail in Figures A2 and A3 in the Appendix.

Here, we applied this technique by using the new unfold toolbox (Ehinger \& Dimigen, 2018), which represents the first-level analysis and provides us with the partial effects (i.e. the beta coefficients or "regression-ERPs", Smith \& Kutas 2015a, 2015b) for each predictor of interest. In a first step, both stimulus onset events and fixation onset events were included as stick functions (also called finite impulse responses, FIR) in the design matrix of the regression model. To account for overlapping activity from adjacent experimental events, the design matrix was then time-expanded in a time window between -300 and +800 ms around each stimulus and fixation onset event. Timeexpansion means that the time points within this window are added as predictors to the regression model. Because the temporal distance between subsequent events in the experiment is variable, it is possible to disentangle their overlapping responses. Time expansion with stick functions is detailed in Ehinger \& Dimigen (2018, p. 8-10) and intuitive illustrations of this step are provided in Serences (2004, their Figure 2) and Ehinger \& Dimigen (2018, their Figure 2).

Using Wilkinson notation, the model formula for scene onset events was defined as:

$\mathrm{ERP} \sim 1+$ Consistency 
In this formula, the beta coefficients for the intercept (1) capture the shape of the overall waveform of the stimulus-ERP in the consistent condition which was used as the reference level, whereas those for Consistency capture the differential effect of presenting an inconsistent scene (relative to a consistent scene) on the ERP. The coefficients for the predictor Consistency therefore correspond to a difference waveform in a traditional ERP analysis (Smith \& Kutas, 2015a, 2015b) and would reveal if semantic processing already occurs as early as during gist processing.

In the same regression model, we also include the onsets of all fixations made on the scene. Fixation onsets were modelled with the formula $\mathrm{FRP} \sim 1$ + Consistency * Type + SaccadeAmplitude

that is, we predicted the FRP for each time-point as a function of the semantic Consistency of the target object (Consistent vs. Inconsistent; Consistent as reference level) in interaction with the Type of fixation (Critical fixation vs. Non-target fixation; Non-target fixation as reference level). In this model, any FRP consistency effects elicited by the pre-target or target fixation would appear as an interaction between Consistency and fixation Type. In addition, we included the incoming Saccade Amplitude (in degrees of visual angle) as a continuous linear covariate to control for the effect of saccade size on the FRP waveform ${ }^{3}$. The regression model was then solved for the betas using MATLAB's glmfit solver (without regularization).

The deconvolution model specified by the two formulas above was run twice: in one version, we treated the pre-target fixation $(t-1)$ as the critical fixation, in the other version the target fixation $(t)$. In a given model, all fixations but the critical ones were defined as non-target fixations. FRPs for fixation $t-1$ and for fixation $t$ were estimated in two separate runs of the model, rather than simultaneously within the same model, because the estimation of overlapping activity was much more stable in this case $^{4}$.

${ }^{3}$ Other low-level variables, such as local image features (e.g., luminance) at the currently foveated image region are also known to modulate the FRP waveform. In the model presented here we did not include these other covariates because (1) their influence on the FRP waveform is small compared to that of saccade amplitude and (2) the properties of the target object (such as its visual saliency) did not differ between conditions (see Materials and Rating). For reasons of simplicity, we included saccade amplitude as a linear predictor of the FRP in the current model, although its influence is non-linear fashion for large saccades (e.g., Dandekar et al., 2011). However, virtually identical results were obtained when we included saccade amplitude instead as a non-linear (spline) predictor (Ehinger \& Dimigen, 2018).

${ }^{4}$ Theoretically, an ideal model would include Type as a three-level predictor, with levels pre-target, target, and non-target fixation. In principle, this would allow us to dissociate which parts of the N400 consistency effects are elicited by fixation $t-1$ versus fixation $t$. However, the practical disadvantage of this approach is that the overlapping activities from both fixation $t$ - 1 and fixation $t$ would then be estimated on comparatively fewer observations (compared to the extremely stable estimate for the numerous non-target fixations). As a consequence, the deconvolution process becomes noisier. By defining either the pre-target fixation or the 
The average number of events entering the model per participant was 65.7 and 73.6 for scene onsets (consistent and inconsistent condition, respectively), 864.2 and 887.9 for non-target fixations $(n t), 58.3$ and 59.8 for pre-target fixations $(t-1)$, and 63.8 and 70.6 for target fixations $(t)$.

Baseline placement for FRPs: Another challenging issue for free viewing EEG experiments is the choice of an appropriate neutral baseline interval for the FRP waveforms (Dimigen et al., 2011; Nikolaev et al., 2016). Baseline placement is particularly relevant for experiments on extrafoveal processing where we do not know in advance when EEG differences will arise and whether they may already develop prior to fixation onset.

For the pre-target fixation $t-1$ and non-target fixations $n t$, we used a standard baseline interval by subtracting the mean channel voltages between -200 and $0 \mathrm{~ms}$ before the event (note that the saccadic spike potential ramping up at the end of this interval was almost completely removed by our ICA procedure; see Figure A1). For fixation $t$, we cannot use such a baseline because by the time the target object is fixated, semantic processing may already be ongoing. Thus, to apply a neutral baseline also to fixation $t$, we subtracted the mean channel voltages in the $200 \mathrm{~ms}$ interval before the preceding fixation $t-1$ also from the FRP aligned to the target fixations $t$ (see Dimigen et al., 2011, Nikolaev et al., 2016, for similar procedures). The scene-onset ERP was corrected with a standard pre-stimulus baseline (-200 to $0 \mathrm{~ms})$.

Group statistics for EEG (second level of analysis): To perform second-level group statistics, averaged EEG waveforms at the single-subject level ("regression-ERPs") were reconstructed from the beta coefficients of the linear deconvolution model. These regression-based ERPs are directly analogous to subject-level averages in a traditional ERP analysis (Smith \& Kutas, 2015a). We then used two complementary statistical approaches to examine consistency effect in the EEG: linear mixed models and a cluster-based permutation test.

LMM in a-priori defined time windows. LMM were used to provide hypothesis-based testing motivated by the previous literature. Specifically, we adopted the spatiotemporal definitions by Võ \& Wolfe (2013) and compared the consistent and inconsistent condition in the time windows from 250 $350 \mathrm{~ms}$ (early effect) and 350 - $600 \mathrm{~ms}$ (late effect) at a mid-central region-of-interest (ROI) of nine electrodes (comprising FC1, FCz, FC2, C1, Cz, C2, CP1, CPz, and CP2; Võ \& Wolfe, 2013). Because the outputs provided by the linear deconvolution model (the first-level analysis) are already aggregated at the level of subject-averages, the only predictor included in these LMMs was the

target fixation as the critical fixation in two separate runs of the model, and by treating all other fixations as non-target fixations, the estimation becomes much more robust. While this means that most overlapping activity is removed, the consistency-specific activity evoked by the pre-target fixation $t$ - 1 (i.e. the N400 effect) will not be removed from the FRP aligned to the subsequent target fixation $t$ and vice versa. 
Consistency of the object (Consistent $=-0.5$, Inconsistent $=0.5)$ with Participant $(24)$ as a random effect.

Cluster-based permutation tests. It is still largely unknown to what extent the timing and topography of traditional ERP effects translates to natural viewing. Therefore, in order to capture the full spatiotemporal dynamics of consistency effects across all channels and time points, we additionally applied the Threshold-Free Cluster Enhancement (TFCE) procedure developed by Smith \& Nichols (2009) and adapted to EEG by Mensen \& Khatami (2013). In a nutshell, TFCE is a nonparametric cluster-based permutation test that controls for multiple comparisons across time and space, while maintaining relatively high sensitivity (compared to a Bonferroni correction). Its advantage over previous cluster permutation tests (e.g., Maris \& Oostenveld, 2007) is that it does not require the experimenter to set arbitrary thresholds (i.e. the cluster-forming threshold). Here we used the MATLAB implementation of TFCE (http://github.com/Mensen/ept_TFCE-matlab) based on $n=$ 2000 random permutations.

Finally, for purely descriptive purposes and to provide a-priori information for future studies, we also plot the $95 \%$ between-subject confidence interval for the consistency effects at the central ROI (corresponding to sample-by-sample paired $t$-testing without correction for multiple comparisons; see also Mudrik et al., 2014) in Figures 4 and 5.

\section{Results}

\section{Eye-movement behaviour}

Figure $3 \mathrm{~A}$ shows the cumulative probability of having fixated the target object as a function of the ordinal number of fixation and semantic consistency, and Table 1 reports the corresponding GLM model coefficients. We found a significant main effect of Consistency; overall, inconsistent objects were looked at with a higher probability than consistent objects. As expected, the cumulative probability of looking at the critical object increased as a function of the Ordinal Number of Fixation. There was also a significant interaction between the two variables.

Complementing this global analysis, we analysed the very first eye-movement during scene exploration to assess whether observers had immediate extrafoveal access to object-scene semantics (Loftus \& Mackworth, 1978). The mean probability of immediate object fixation was $12.77 \%$, and we observed a numeric advantage of inconsistent objects over consistent objects (Figure 3B) which failed to reach significance (Table 2). The latency to first fixation on the target object is another measure to capture the potency of an object in attracting early attention in extrafoveal vision (e.g., Underwood \& Foulsham, 2006; Võ \& Henderson, 2009). This measure is defined as the time elapsed between the onset of the scene image and the first fixation on the critical object. Importantly, this latency was significantly shorter for inconsistent objects (Figure 3C, Table 2). 
Figure 3

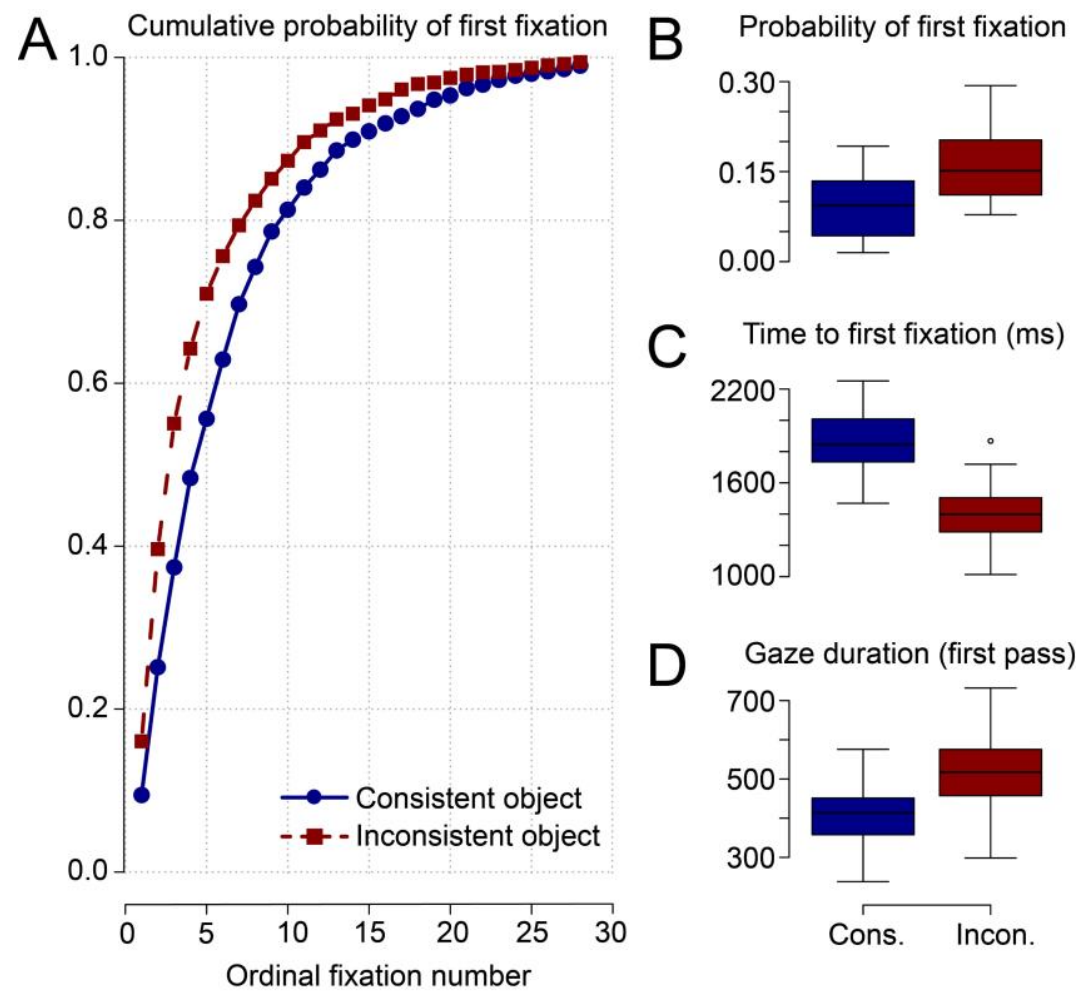

Figure 3. Eye-movement correlates of early overt attention towards consistent and inconsistent critical objects. A. Cumulative probability of fixating at the critical object as a function of the ordinal fixation number on the scene. Blue solid line $=$ consistent object; red dashed line $=$ inconsistent object. $\mathbf{B}$. Probability of fixating at the critical object already at the first fixation after scene onset. C. Latency until fixating the critical object for the first time. D. First-pass gaze duration for the critical object, i.e. the sum of all fixation durations from first entry to first exit.

Table 1

Predictor

Cumulative probability of First Fixation

\begin{tabular}{|c|c|c|c|c|}
\hline & $\beta$ & $\overline{S E}$ & $z$-value & $\operatorname{Pr}(>|z|)$ \\
\hline Intercept & -1.04 & 0.02 & -49.91 & 0.00001 \\
\hline Nr. Fixation & -2.00 & 0.05 & -35.9 & 0.00001 \\
\hline Consistency & 0.17 & 0.03 & 5.8 & 0.00001 \\
\hline Consistency $\times$ Nr. Fixation & -0.71 & 0.09 & -7.9 & 0.00001 \\
\hline
\end{tabular}

Table 1. Cumulative probability of having fixated the critical object as a function of the ordinal number of fixations on the scene (binomial probit). The centred predictors are Consistency (Consistent: -0.5, Inconsistent: 0.5) and Number of Fixation 
Moreover, we analysed gaze duration as a measure of foveal object processing time (e.g., Henderson et al., 1999). First-pass gaze duration for a critical object is defined as the sum of all fixation durations from first entry to first exit. On average, participants looked longer at inconsistent (520.2 ms) than consistent objects (408.9 ms) before leaving the target object for the first time, and this difference was significant (Table 2). Table 3 summarizes some additional oculomotor characteristics in the different conditions.

Table 2

\begin{tabular}{|c|c|c|c|c|c|c|c|c|c|}
\hline \multirow[b]{2}{*}{ Predictor } & \multicolumn{3}{|c|}{$\begin{array}{l}\text { Probability } \\
\text { of first fixation }\end{array}$} & \multicolumn{3}{|c|}{ Time to first fixation } & \multicolumn{3}{|c|}{ Gaze Duration } \\
\hline & $\beta$ & $S E$ & $z$ & $\beta$ & $S E$ & $t$ & $\beta$ & $S E$ & $t$ \\
\hline Intercept & -2.93 & 0.19 & $-14.73^{* * * *}$ & $1,904.4$ & 83.8 & $22.7^{* * * *}$ & 400.1 & 20.97 & $19.08^{* * * *}$ \\
\hline Consistency & 0.21 & 0.15 & 1.38 & -246.4 & 64.0 & $-3.85^{* * * *}$ & 105.0 & 20.77 & $7.08^{* * * *}$ \\
\hline
\end{tabular}

Table 2. Probability and time to first fixation. The dummy coded predictor is Consistency (with consistent object as the reference level). We report the $\beta$, the standard error, the $z$ - (for binomial link) and $t$-value. Asterisks indicate significant predictors.

Table 3

\begin{tabular}{lcc} 
& Consistent & Inconsistent \\
& Mean \pm SD & Mean \pm SD \\
\hline Fixation duration $(t-2)$, in ms & $226 \pm 108$ & $219 \pm 98$ \\
Fixation duration $(t-1)$ & $212 \pm 98$ & $202 \pm 95$ \\
Fixation duration $(t)$ & $268 \pm 151$ & $270 \pm 140$ \\
Fixation duration $(t+1)$ & $244 \pm 121$ & $261 \pm 135$ \\
Incoming saccade amplitude to $t-1\left(^{\circ}\right)$ & $6.1 \pm 5.2$ & $6.0 \pm 5.0$ \\
Incoming saccade amplitude to $t\left(^{\circ}\right)$ & $8.6 \pm 5.3$ & $8.3 \pm 4.9$ \\
Ordinal number of first fixation on the target & $6.8 \pm 6.1$ & $5.2 \pm 5.4$ \\
\hline
\end{tabular}

Table 3. Mean and standard deviation of different oculomotor covariates for scenes with semantically consistent and inconsistent objects. Fixation $t+1$ is the first fixation after leaving the region of the target object. 
Table 4

\begin{tabular}{|c|c|c|c|c|}
\hline Type of Event & Analysis window & $\beta$ & $S E$ & $t$-value \\
\hline \multirow{2}{*}{ Scene onset } & Early $(250-350 \mathrm{~ms})$ & 0.28 & 0.39 & 0.71 \\
\hline & Late $(350-600 \mathrm{~ms})$ & 0.34 & 0.39 & 0.37 \\
\hline \multirow[b]{2}{*}{$n t$} & Early $(250-350 \mathrm{~ms})$ & -0.05 & 0.07 & -0.71 \\
\hline & Late $(350-600 \mathrm{~ms})$ & -0.09 & 0.08 & -1.10 \\
\hline \multirow[b]{2}{*}{$t-1$} & Early (250-350 ms) & -0.28 & 0.15 & $-1.77(*)$ \\
\hline & Late $(350-600 \mathrm{~ms})$ & -0.46 & 0.12 & $-3.76 * * *$ \\
\hline \multirow[b]{2}{*}{$t$} & Early $(250-350 \mathrm{~ms})$ & -0.52 & 0.17 & $-3.03 * *$ \\
\hline & Late $(350-600 \mathrm{~ms})$ & -0.38 & 0.15 & $-2.43 *$ \\
\hline$t$ & Early $(250-350 \mathrm{~ms})$ & -0.34 & 0.16 & $-2.20 *$ \\
\hline $\begin{array}{l}\text { (control analysis with baseline } \\
\text { before fixation } t \text { ) }\end{array}$ & Late $(350-600 \mathrm{~ms})$ & -0.20 & 0.17 & -1.14 \\
\hline
\end{tabular}

Table 4. Mixed-effects models with maximal random structure for the FRP at the mid-central region-of-interest (comprising electrodes $\mathrm{FC} 1, \mathrm{FCz}, \mathrm{FC} 2, \mathrm{C} 1, \mathrm{Cz}, \mathrm{C} 2, \mathrm{CP} 1, \mathrm{CPz}$, and $\mathrm{CP} 2$ ) for two temporal windows of analysis (Early, 250-350 ms and Late, 350-600 ms) as predicted by Consistency (Consistent $=-0.5$, Inconsistent $=0.5$ ) of which we report the $\beta$ the standard error, and the $t$-value. Asterisks indicate the level of significance.

\section{Electrophysiological results}

Figures 4 and 5 depict the ERP evoked by the presentation of the scene as well as the FRPs for the three types of fixation analysed. Results focus on the mid-central ROI for which effects of objectscene consistency have been previously reported. Waveforms for other scalp sites are depicted in Figures A4 to A7 in the Appendix.

Scene-onset ERP. The left panels of Figure 4 show the grand-average ERP aligned to scene onset. Although inspection of the scalp maps indicated slightly more positive amplitudes over central right-hemispheric electrodes in the inconsistent condition, these differences were not statistically significant. Specifically, no effect of Consistency was found with the LMM analysis in the early or the late time window (see Table 4 for detailed LMM results). Similarly, the TFCE test across all channels and time points yielded no significant Consistency effect (all $p$-values $>0.2$, see Figure 4D). Thus, the semantic consistency of the target object did not influence the neural response to the initial presentation of the scene.

Non-target fixation, $\boldsymbol{n t}$. Next, we tested whether fixations on scenes with an inconsistent object evoke a globally different neural response than those on scenes containing a consistent object. As the 
right panels of Figure 4 show, this was not the case: Consistency had no effect on the FRP for nontarget ( $n t$ ) fixations, neither in the LMM analysis (see Table 4) nor in the TFCE statistic (all $p$-values $>0.2$, see Figure $4 \mathrm{H})$.

Figure 4
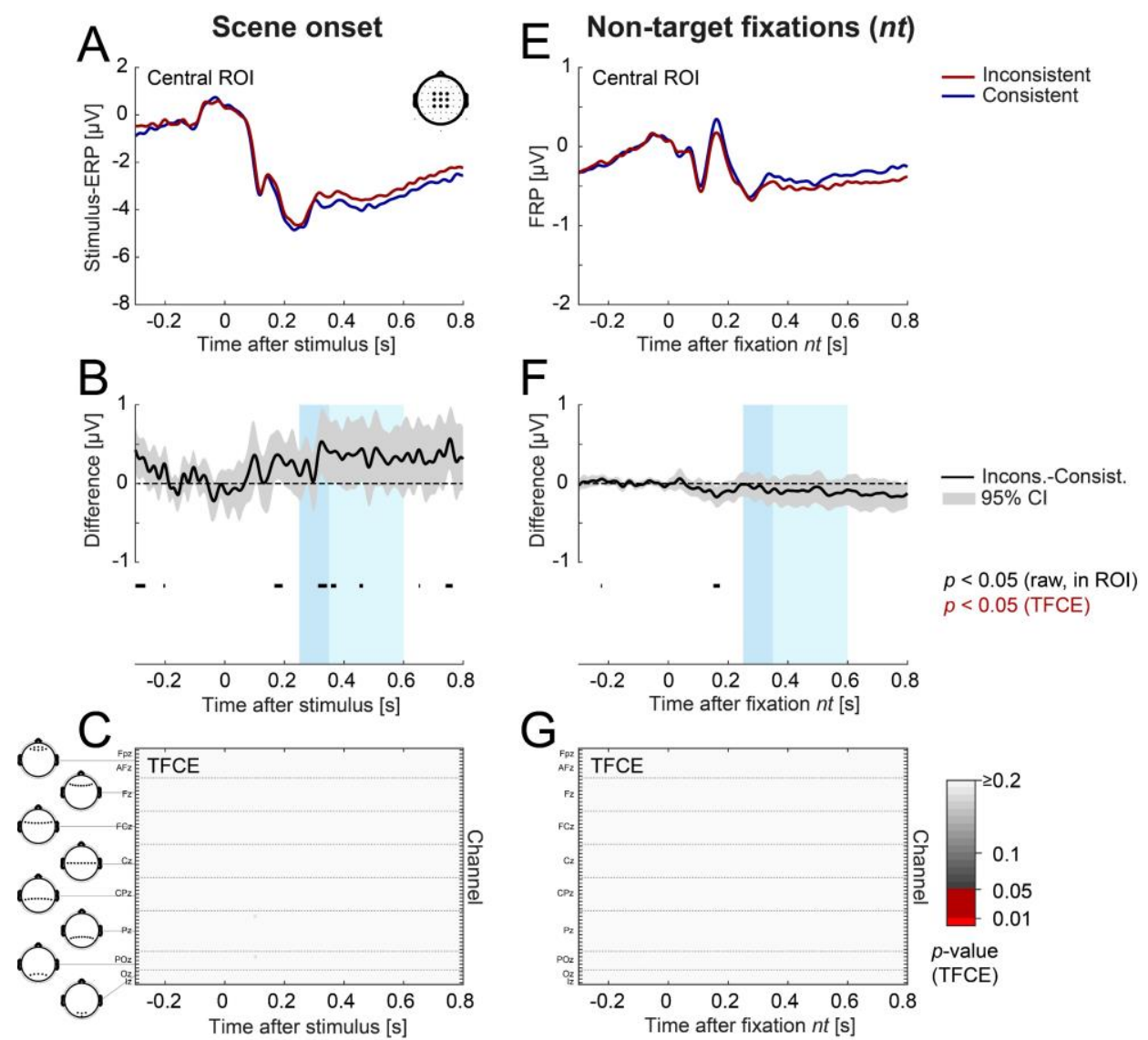

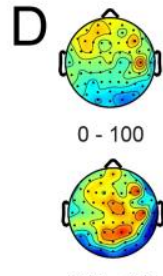

$300-400$

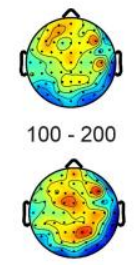

$400-500$

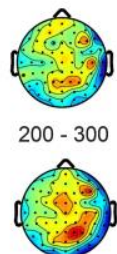

$500-600[\mathrm{~ms}]$
$\mathrm{H}$

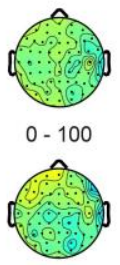

$300-400$

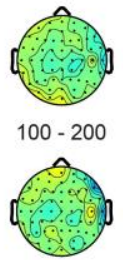

$400-500$

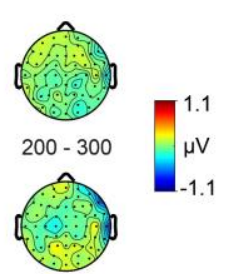

$500-600[\mathrm{~ms}]$

Figure 4. Stimulus-ERP aligned to scene onset (left panels) and FRP aligned to non-target fixations (right panels) as a function of object-scene consistency. A and E. Grand-average ERP/FRP at the central region-of-interest (comprising electrodes FC1, FCz, FC2, C1, Cz, C2, CP1, CPz, CP2). Red lines represent the Inconsistent condition, blue lines represent the Consistent condition. $\mathbf{B}$ and F. Corresponding difference waves (inconsistent minus consistent) at the central ROI. Grey shading illustrates the $95 \%$ confidence interval (without correction for multiple comparisons) of the difference wave with values outside the CI also marked in black below the curve. The two windows used for LMM statistics (250-350 and 350-600 ms) are indicated in light blue. C and G. Results of clusterbased permutation testing (TFCE) across all electrodes/time points. D and H. Scalp topographies of the consistency effect (inconsistent minus consistent) averaged across successive $100 \mathrm{~ms}$ time windows. Object-scene consistency had no significant effects on the stimulus-ERP or on the FRP elicited by non-target fixations, neither in the LMM statistic, nor in the cluster-based permutation test. 
Figure 5

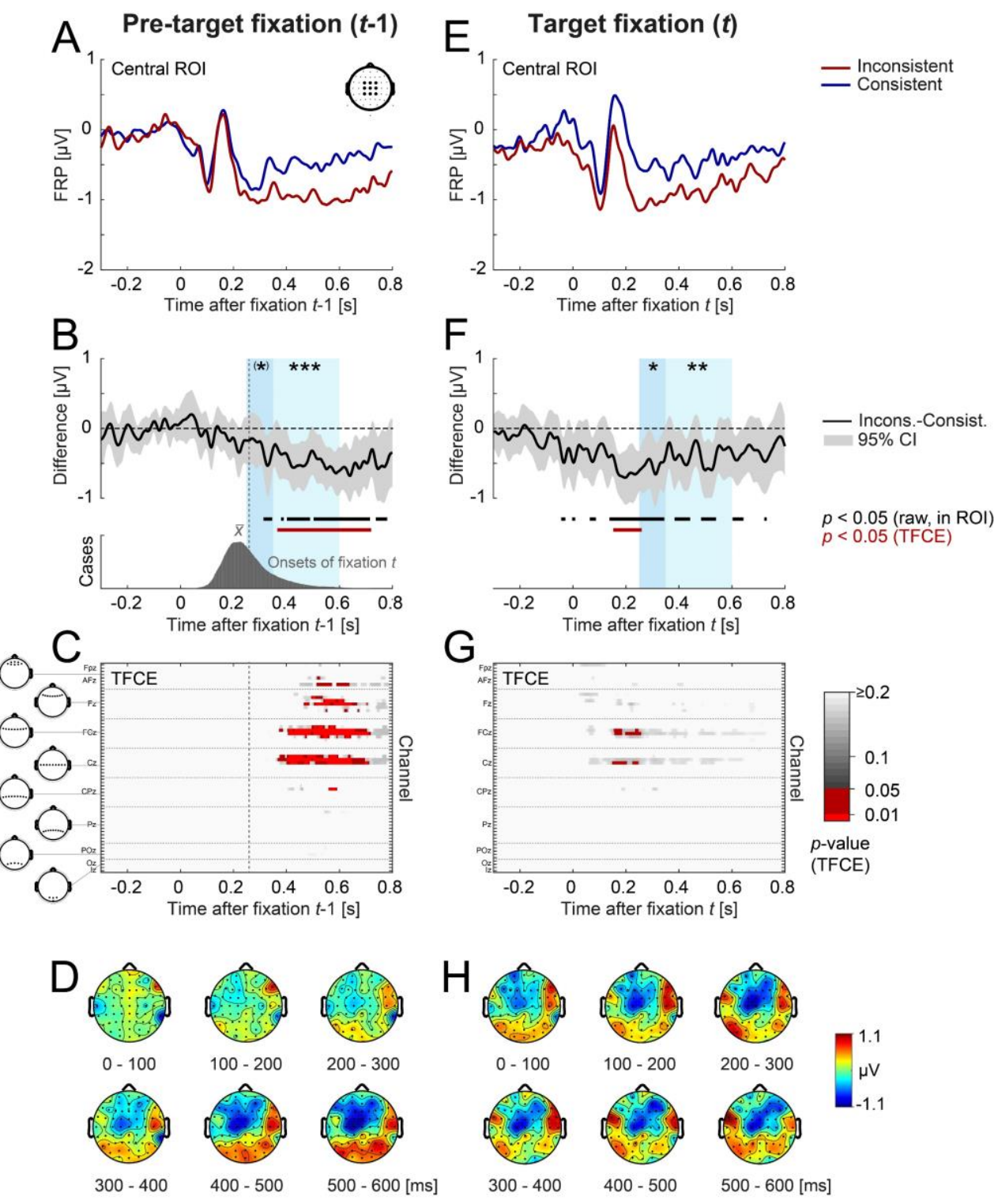

Figure 5. Grand-average FRP elicited by pre-target fixation (left panels) and target fixation (right panels) as a function of object-scene consistency. (A, E) Grand-average FRPs at the central ROI. (B, F) Difference waves at the central ROI. In panel B, the grey distribution shows the onset of fixation $t$ relative to the onset of the pre-target fixation $t-1$, with the vertical dotted line indicating the mean latency $(260 \mathrm{~ms})$. (C, G) Results of cluster-based permutation testing (TFCE). Significant $(p<0.05)$ channels and time points are marked in red. The time intervals with a significant effect in the TFCE statistic are also illustrated by the red bars in panels B and F. (D, H) Scalp topographies of the consistency effect (inconsistent minus consistent) across successive $100 \mathrm{~ms}$ time windows. A frontocentral N400 effect emerged in the FRP time-locked to fixation $t-1$ and reached significance shortly after the eyes had moved on to fixation $t$. This effect then continued during fixation $t$ reaching a maximum $200 \mathrm{~ms}$ after the start of the target fixation.

Pre-target fixation, $t$-1. Figure 5 depicts the FRPs aligned to the pre-target and target fixations. Importantly, in the FRP aligned to the pre-target fixation $t$-1, waveforms began to clearly diverge between the two consistency conditions, developing into a long-lasting fronto-central negativity in the 
inconsistent as compared to the consistent condition (Figure 5A and 5B; see also Figure A6). The scalp distribution of this difference, shown in Figure 6, closely resembled the frontocentral N400 (and N300) previously reported in ERP studies on object-scene consistency (e.g., Mudrik et al., 2014; Võ $\&$ Wolfe, 2013). In the LMM analyses conducted on the mid-central ROI, this effect was marginally significant $(p<0.1)$ for the early time window ( 250 to $350 \mathrm{~ms}$ ), but became highly significant between 350 and $600 \mathrm{~ms}(p<0.001$, Table 4). The TFCE test across all channels and time points revealed significant differences $(p<0.05$ ) between $372 \mathrm{~ms}$ and $721 \mathrm{~ms}$ after fixation onset in a cluster of 14 frontocentral electrodes that was shifted slightly to the left hemisphere. Thus, we were able to measure a significant frontocentral N400 modulation during natural scene viewing and this effect already emerged in FRPs aligned to the pre-target fixation.

On average, the target fixation $t$ occurred $260 \mathrm{~ms}$ after fixation $t-1$, as marked by the vertical dashed line in Figure 5B. This means that, on average, at the time when the ERP consistency effect reached significance (estimated at $372 \mathrm{~ms}$ using the conservative TFCE procedure), the eyes had been looking at the target object for only $112 \mathrm{~ms}$ (372 minus $260 \mathrm{~ms}$ ).

Target fixation, $t$. An anterior N400 effect was also clearly visible in the FRP aligned to fixation $t$. In the LMM analysis at the central ROI, the effect was significant in both the early (250-350 ms, $p<$ 0.01 ) and late window (350-600 ms, $p<0.05$; see Table 4). However, compared to the effect aligned to the pre-target fixation, this $\mathrm{N} 400$ was significant at only a few electrodes in the TFCE statistic $(\mathrm{Cz}$, $\mathrm{FCz}$, and FC1; see Figure 6). Aligned to the target fixation $t$, the N400 also peaked extremely early. As Figure 5D shows, a frontocentral negativity was qualitatively already visible within the first 100 ms after fixation onset. In the TFCE statistic, it then became significant in a brief interval between 151 and $263 \mathrm{~ms}$ after fixation onset, during which the N400 effect also reached its peak.

Figure 5E shows that voltages at the central ROI were numerically already more negative in the inconsistent condition during the baseline interval, that is, before the critical object was fixated. To understand the role of activity already present before fixation onset, we repeated the FRP analyses for fixation $t$ after applying a standard baseline correction, with the baseline placed immediately before the target fixation itself (-200 to $0 \mathrm{~ms}$ ). In this way, we eliminate any weak N400-like effects that may have already been ongoing before fixation onset. Interestingly, in the resulting FRP waveforms, the target-locked N400 effects were weakened: The N400 effect now failed to reach significance in the TFCE statistic and in the LMM analysis for the second window (350 to $600 \mathrm{~ms}$; see last row of Table 4) and only remained significant for the early window (250 to $350 \mathrm{~ms}$ ). This indicates that some N400-like negativity was already ongoing before target fixation onset. Taken together, our results therefore suggest that consistency effects emerged around or very shortly after target fixation onset. 


\section{Discussion}

Figure 6

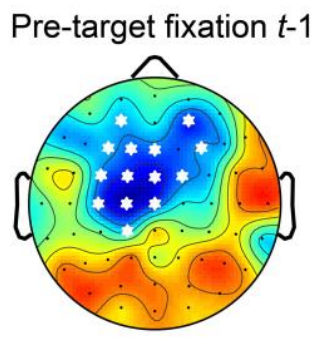

Inconsist. - Consist.

372 to $721 \mathrm{~ms}$

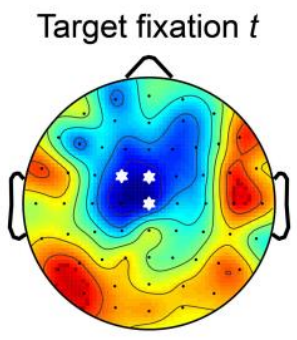

Inconsist. - Consist.

151 to $263 \mathrm{~ms}$

Figure 6. Scalp distribution of frontocentral N400 effects in the time windows significant in the TFCE statistic (see also Figure 5). White asterisks highlight electrodes with a significant consistency $(p<0.05)$ effect. In the FRP aligned to the pre-target fixation (left), a consistency effect was observed between 372 to $721 \mathrm{~ms}$ at 13 frontocentral channels. In the FRP aligned to the target fixation (right), an effect was observed between 151 and $263 \mathrm{~ms}$ at three frontocentral channels.

Substantial research in vision science has been devoted to understanding the behavioural and neural mechanisms underlying object recognition (e.g., Biederman, 1972; Loftus \& Mackworth, 1978, for seminal work on the topic). At the core of this debate are the type of object features that are accessed (e.g., low-level vs. high-level), the time-course of their processing (e.g., pre-attentive vs. attentive), and the region of the visual field in which these features can be acquired (e.g., foveal vs. extrafoveal). A particularly controversial topic is how and when objects are processed that are (in)consistent with the overall meaning of the scene within which they occur.

In the current study, we approached these questions from a new perspective by co-registering eye-movements and EEG while participants freely inspected images of real-world scenes in which a critical object was either consistent or inconsistent with the scene context. As a novel finding, we demonstrate a fixation-related N400 effect during natural scene viewing. Moreover, behavioural and electrophysiological measures provide converging evidence that the extraction of object-scene semantics can already begin in extrafoveal vision, before the critical object is fixated.

It is a rather undisputed finding that inconsistent objects, such as a flashlight in a bathroom, require increased processing when selected as targets of overt attention. Accordingly, several eyemovement studies have reported longer gaze durations on inconsistent than consistent objects, probably reflecting the greater effort required to resolve the conflict between object meaning and scene context (e.g., Cornelissen \& Võ, 2017; De Graef et al., 1990; Henderson et al., 1999). In addition, a number of traditional ERP studies using steady-fixation paradigms have found that inconsistent objects elicit a larger negative brain response over frontocentral channels (an N300/N400 complex) as compared to consistent objects.

However, previous research with eye-movements remained inconclusive on whether semantic processing can take place prior to foveal inspection of the object. Evidence in favour of extrafoveal 
processing of object-scene semantics comes from studies in which inconsistent objects were selected for fixation earlier than consistent ones (e.g., LaPointe \& Milliken, 2016; Mackworth \& Morandi, 1967; Underwood et al., 2008). However, other studies have not found evidence for earlier selection of inconsistent objects (e.g., De Graef et al., 1990; Henderson et al., 1999; Võ \& Henderson, 2009, 2011). Extrafoveal and peripheral vision are known to be crucial for saccadic programming (e.g., Nuthmann, 2014). Therefore, any demonstration that semantic information can act as a source of guidance for fixation selection in scenes implies that some semantic processing must have occurred prior to its fixation (i.e., in extrafoveal vision).

ERPs are highly sensitive to semantic processing (Kutas \& Federmeier, 2011) and provide excellent temporal resolution to investigate the time course of object processing. However, an obvious limitation of all existing ERP studies is that observers were not allowed to explore the scene with saccades; severely constraining their normal attentional dynamics. Instead, the critical object was usually large and/or placed near the point of fixation. Hence, these studies were unable to establish whether semantic processing can take place prior to its foveal inspection.

In the current study, we addressed this problem by recording behavioural and brain-electric correlates of object processing simultaneously. Specifically, we analysed different eye-movement responses that tap into extrafoveal and foveal processing along with FRPs time-locked to the first fixation of the critical object $(t)$ and the fixation preceding it $(t-1)$. We also analysed the scene-onset ERP evoked by the trial-initial presentation of the image. Recent advances in linear deconvolution methods for EEG (e.g., Ehinger \& Dimigen, 2018) allowed us to disentangle the overlapping brain potentials produced by the scene onset and the subsequent fixations and to control for the modulating influence of saccade amplitude on the FRP.

In eye-movement behaviour, we found evidence for hypothesis B as outlined in the Introduction. The probability of fixating the target object immediately after the first saccade was rather low (12.8\%) and not affected by Consistency. This speaks against hypothesis A. However, in agreement with some previous studies, the results for the latency to first fixation of the critical object show that inconsistent objects were on average looked at sooner than consistent objects, providing evidence for the prioritization of scene-inconsistent objects via extrafoveal processing of semantic features. At the same time, we observed longer gaze durations on inconsistent objects, replicating previous findings. Thus, we found evidence for the extrafoveal processing of object-scene (in)consistencies, but also differences in the subsequent foveal processing.

The corresponding FRP waveforms showed a negative shift over frontal and central scalp sites when participants fixated a scene-inconsistent object. This result is in agreement with traditional ERP studies that have shown an frontocentral N300/N400 complex after passive foveal stimulation (e.g., Coco et al., 2017; Ganis \& Kutas, 2003; Mudrik et al., 2014; Võ \& Wolfe, 2013) and extends this finding for the first time to a natural viewing situation with eye-movements. Importantly, the time 
course of this effect suggests that it was already initiated during the preceding fixation $(t-1)$, but then carried on through fixation $(t)$ on the target object.

As a cautionary note, it should be said that it is not trivial to unambiguously ascribe typical N400 (and N300) effects in the EEG to either extrafoveal or foveal processing. The reason is that these canonical congruency effects only begin 200-250 ms after stimulus onset (Draschkow et al., 2018; Mudrik et al., 2010). This means that even a purely extrafoveal effect would be almost impossible to measure during the pre-target fixation $(t-1)$ itself, since it would only emerge at a time when the eyes are already moving to the target object. That being said, three properties of the observed FRP consistency effect suggest that it was already initiated during the pre-target fixation:

First, due to the temporal jitter introduced by variable fixation durations, an effect that only arises in foveal vision should be the most robust in the FRP averages aligned to fixation $t$, but latencyjittered and attenuated in those aligned to fixation $t-1$. However, the opposite was the case: A significant frontocentral N400 effect was seen at more electrodes and for longer time intervals in the FRP aligned to the pre-target fixation as compared to the actual target fixation. The second argument for extrafoveal contributions to the effect is the forward-shift in its time course. Relative to fixation $t$, the observed N400 occurred almost immediately: As the effect topographies in Figure 5E show, the frontocentral negativity for inconsistent objects was qualitatively visible within the first $100 \mathrm{~ms}$ after fixation onset. Using the conservative TFCE statistic, the effect then became significance at $151 \mathrm{~ms}$ and reached its peak after $200 \mathrm{~ms}$, much earlier than what we would expect from the canonical N300 or N400 effect elicited by foveal stimulation. Finally, we found that this consistency effect aligned to the target fixation $(t)$ became weaker (and non-significant in two out of the three statistical measures considered) if the baseline interval for FRP analysis was placed directly before this fixation. This indicates that at least a weak frontocentral negativity in the inconsistent condition was already present during the baseline period before the target was fixated. Together, these results are difficult to reconcile with a pure foveal processing account and suggest instead that semantic processing of the object was at least initiated in extrafoveal vision (and then continued after it was foveated).

Crucially, we did not find any effect of target consistency in the traditional ERP aligned to scene onset. In line with the behavioural results, this goes against the most extreme hypothesis A postulating that object semantics can be extracted from peripheral vision already at the first glance of a scene (Loftus \& Mackworth, 1978). Similarly, there was no effect of consistency on the FRPs evoked by the non-target fixations, an important finding that shows that the effects of consistency found in our study are specific to fixations associated with the target object.

Taken together, our behaviour and electrophysiological findings are consistent with the claim formulated in hypothesis B that objects can be recognized outside of the fovea or even in the visual periphery, at least to some degree. The fact that the average amplitude of the saccade that first entered the critical object was more than $8^{\circ}$ in our study (see Table 4) also indicates that this processing at a 
semantic level is possible over a fairly large area of the visual field. In principle, this result is in agreement with the idea that object-scene integration may be happening in a parallelized fashion (cf. De Cesarei \& Loftus, 2011; Weidner \& Müller, 2013; Herwig \& Schneider, 2014; Buetti, Cronin, Madison, Wang, \& Lleras, 2016). Indirectly, our results also speak about the unit of saccade targeting and, by inference, attentional selection during scene viewing, since semantic influences on eye guidance suggest an object- and meaning-based, rather than image-based guidance of attention (e.g., Hwang, Wang, \& Pomplun, 2011; Henderson \& Hayes, 2017).

In sum, our findings converge to suggest that the visual system is capable of accessing semantic features of objects in extrafoveal vision to guide attention towards objects that do not fit to the scene's overall meaning. They also highlight the utility of investigating attentional and neural mechanisms in parallel to uncover the mechanisms underlying object recognition during the unconstrained exploration of naturalistic scenes.

\section{References}

Antes, J. R. (1974). The time course of picture viewing. Journal of Experimental Psychology, 103(1), 62-70. https://doi.org/10.1037/h0036799

Bates, D., Mächler, M., Bolker, B., \& Walker, S. (2015). Fitting Linear Mixed-Effects Models using lme4. Journal of Statistical Software, 67(1), 1-48. https://doi.org/10.18637/jss.v067.i01

Belke, E., Humphreys, G. W., Watson, Derrick, G., Meyer, A. S., \& Telling, A. L. (2008). Top-down effects of semantic knowledge in visual search are modulated by cognitive but not perceptual load. Perception \& Psychophysics, 70(8), 1444-1458. https://doi.org/10.3758/PP.70.8.1444

Berg, P., \& Scherg, M. (1994). A multiple source approach to the correction of eye artifacts. Electroencephalography and Clinical Neurophysiology, 90(3), 229-241. https://doi.org/10.1016/0013-4694(94)90094-9

Biederman, I. (1972). Perceiving real-world scenes. Science, 177, 77-80. https://doi.org/10.1126/science.177.4043.77

Bonitz, V. S., \& Gordon, R. D. (2008). Attention to smoking-related and incongruous objects during scene viewing. Acta Psychologica, 129(2), 255-263. https://doi.org/10.1016/j.actpsy.2008.08.006

Brouwer, A.-M., Reuderink, B., Vincent, J., van Gerven, M. A. J., \& van Erp, J. B. F. (2013). Distinguishing between target and nontarget fixations in a visual search task using fixation-related potentials. Journal of Vision, 13(3), :17, 1-17. https://doi.org/10.1167/13.3.17

Buetti, S., Cronin, D. A., Madison, A. M., Wang, Z., \& Lleras, A. (2016). Towards a Better Understanding of Parallel Visual Processing in Human Vision: Evidence for Exhaustive Analysis of Visual Information. Journal of Experimental Psychology: General, 145(6), 672-707. https://doi.org/10.1037/xge0000163

Coco, M. I., Araujo, S., \& Petersson, K. M. (2017). Disentangling stimulus plausibility and contextual congruency: Electro-physiological evidence for differential cognitive dynamics. Neuropsychologia, 96, 150-163. https://doi.org/10.1016/j.neuropsychologia.2016.12.008

Cornelissen, T. H. W., \& Võ, M. L.-H. (2017). Stuck on semantics: Processing of irrelevant object-scene inconsistencies modulates ongoing gaze behavior. Attention, Perception, \& Psychophysics, 79(1), 154-168. https://doi.org/10.3758/s13414-016-1203-7

Dandekar, S., Privitera, C., Carney, T., \& Klein, S. A. (2011). Neural saccadic response estimation during natural viewing. Journal of Neurophysiology, 107(6), 1776-1790. https://doi.org/10.1152/jn.00237.2011

Davenport, J. L., \& Potter, M. C. (2004). Scene consistency in object and background perception. Psychological Science, 15(8), 559-564. https://doi.org/10.1111/j.0956-7976.2004.00719.x

De Cesarei, A., \& Loftus, G. R. (2011). Global and local vision in natural scene identification. Psychonomic Bulletin \& Review, 18, 840-847. https://doi.org/10.3758/s13423-011-0133-6

De Graef, P., Christiaens, D., \& D’Ydewalle, G. (1990). Perceptual effects of scene context on object identification. Psychological Research, 52(4), 317-329. https://doi.org/10.1007/BF00868064

Delorme, A., \& Makeig, S. (2004). EEGLAB: an open sorce toolbox for analysis of single-trail EEG dynamics including independent component analysis. Journal of Neuroscience Methods, 134, 9-21. https://doi.org/10.1016/j.jneumeth.2003.10.009 
Devillez, H., Guyader, N., \& Guerin-Dugue, A. (2015). An eye fixation-related potentials analysis of the P300 potential for fixations onto a target object when exploring natural scenes. Journal of Vision, 15(13);20 1-31. https://doi.org/10.1167/15.13.20

Dimigen, O. (2018). Optimized ICA-based removal of ocular EEG artifacts from free viewing experiments. BioRxiv, (0), 446955. https://doi.org/10.1101/446955

Dimigen, O., Sommer, W., Hohlfeld, A., Jacobs, A. M., \& Kliegl, R. (2011). Co-Registration of Eye Movements and EEG in Natural Reading: Analyses and Review. Journal of Experimental Psychology: General, 140(4), 552572. https://doi.org/10.1037/a0023885

Draschkow, D., Heikel, E., Võ, M. L.-H., Fiebach, C. J., \& Sassenhagen, J. (2018). No evidence from MVPA for different processes underlying the N300 and N400 incongruity effects in object-scene processing. Neuropsychologia, 120(July), 9-17. https://doi.org/10.1016/J.NEUROPSYCHOLOGIA.2018.09.016

Dyck, M., \& Brodeur, M. B. (2015). ERP evidence for the influence of scene context on the recognition of ambiguous and unambiguous objects. Neuropsychologia, 72, 43-51. https://doi.org/10.1016/j.neuropsychologia.2015.04.023

Ehinger, B., \& Dimigen, O. (2018). Unfold: An integrated toolbox for overlap correction, non-linear modeling, and regression-based EEG analysis. BioRxiv, 360156. https://doi.org/10.1101/360156

Feldman, J. (2003). What is a visual object? Trends in Cognitive Sciences, 7(6), 252-256. https://doi.org/10.1016/S1364-6613(03)00111-6

Fenske, M. J., Aminoff, E., Gronau, N., \& Bar, M. (2006). Top-down facilitation of visual object recognition: objectbased and context-based contributions. Progress in Brain Research, 155 B, 3-21. https://doi.org/10.1016/S0079-6123(06)55001-0

Ganis, G., \& Kutas, M. (2003). An electrophysiological study of scene effects on object identification. Cognitive Brain Research, 16(2), 123-144. https://doi.org/10.1016/S0926-6410(02)00244-6

Garcia-Diaz, A., Fdez-Vidal, X. R., Pardo, X. M., \& Dosil, R. (2012). Saliency from hierarchical adaptation through decorrelation and variance normalization. Image and Vision Computing, 30(1), 51-64. https://doi.org/10.1016/j.imavis.2011.11.007

Henderson, J. M., \& Hayes, T. R. (2017). Meaning-based guidance of attention in scenes as revealed by meaning maps. Nature Human Behaviour, 1(10), 743-747. https://doi.org/10.1038/s41562-017-0208-0

Henderson, J. M., Weeks, Jr, P. A., \& Hollingworth, A. (1999). The effects of semantic consistency on eye movements during complex scene viewing. Journal of Experimental Psychology: Human Perception and Performance, 25(1), 210-228. https://doi.org/10.1037//0096-1523.25.1.210

Herwig, A., \& Schneider, W. X. (2014). Predicting object features across saccades: Evidence from object recognition and visual search. Journal of Experimental Psychology: General, 143(5), 1903-1922. https://doi.org/10.1037/a0036781

Hwang, A. D., Wang, H. C., \& Pomplun, M. (2011). Semantic guidance of eye movements in real-world scenes. Vision Research, 51(10), 1192-1205. https://doi.org/10.1016/j.visres.2011.03.010

Itti, L., Koch, C., \& Niebur, E. (1998). A Model of Saliency-Based Visual Attention for Rapid Scene Analysis. IEEE Transaction on Pattern Analysis and Machine Learning, 20(11), 1254-1259. https://doi.org/10.1109/TPAMI.2012.125

Jung, T.-P., Humphries, C., Lee, T.-W., Makeig, S., McKeown, M. J., Iragui, V., \& Sejnowski, T. J. (1998). Extended ICA removes artifacts from electroencephalographic recordings. Advances in Neural Information Processing Systems, 10, 894-900.

Kamienkowski, J. E., Ison, M. J., Quiroga, R. Q., \& Sigman, M. (2012). Fixation-related potentials in visual search: A combined EEG and eye tracking study. Journal of Vision, 12(7), :4, 1-20. https://doi.org/10.1167/12.7.4

Kaunitz, L. N., Kamienkowski, J. E., Varatharajah, A., Sigman, M., Quiroga, R. Q., \& Ison, M. J. (2014). Looking for a face in the crowd: Fixation-related potentials in an eye-movement visual search task. NeuroImage, 89, 297305. https://doi.org/10.1016/j.neuroimage.2013.12.006

Kliegl, R., Dambacher, M., Dimigen, O., Jacobs, A. M., \& Sommer, W. (2012). Eye movements and brain electric potentials during reading. Psychological Research. https://doi.org/10.1007/s00426-011-0376-X

Kretzschmar, F., Bornkessel-Schlesewsky, I., \& Schlesewsky, M. (2009). Parafoveal versus foveal N400s dissociate spreading activation from contextual fit. NeuroReport, 20(18), 1613-1618. https://doi.org/10.1097/WNR.0b013e328332c4f4

Kristensen, E., Guerin-Dugué, A., \& Rivet, B. (2017). Regularization and a general linear model for event-related potential estimation. Behavior Research Methods, 49(6), 2255-2274. https://doi.org/10.3758/s13428-017-0856Z

Kristensen, E., Rivet, B., \& Guerin-Dugué, A. (2017). Estimation of overlapped Eye Fixation Related Potentials: The General Linear Model, a more flexible framework than the ADJAR algorithm. Journal of Eye Movement Research, 10(1), 1-27. https://doi.org/http://dx.doi.org/10.16910/jemr.10.1.7 
Kutas, M., \& Federmeier, K. D. (2011). Thirty years and counting: finding meaning in the N400 component of the event-related brain potential (ERP). Annual Review of Psychology, 62(1), 621-647. https://doi.org/10.1146/annurev.psych.093008.131123

LaPointe, M. R. P., \& Milliken, B. (2016). Semantically incongruent objects attract eye gaze when viewing scenes for change. Visual Cognition, 24(1), 63-77. https://doi.org/10.1080/13506285.2016.1185070

Loftus, G. R., \& Mackworth, N. H. (1978). Cognitive determinants of fixation location during picture viewing. Journal of Experimental Psychology. Human Perception and Performance, 4(4), 565-572. https://doi.org/10.1037/0096-1523.4.4.565

Mackworth, N. H., \& Morandi, A. J. (1967). The gaze selects information details within picutres. Perception and Psychophysics, 2(11), 547-552. https://doi.org/10.3758/BF03210264

Maris, E., \& Oostenveld, R. (2007). Nonparametric statistical testing of EEG- and MEG-data. Journal of Neuroscience Methods, 164(1), 177-190. https://doi.org/10.1016/j.jneumeth.2007.03.024

Mensen, A., \& Khatami, R. (2013). Advanced EEG analysis using threshold-free cluster-enhancement and nonparametric statistics. NeuroImage, 67, 111-118. https://doi.org/10.1016/j.neuroimage.2012.10.027

Moores, E., Laiti, L., \& Chelazzi, L. (2003). Associative knowledge controls deployment of visual selective attention. Nature Neuroscience, 6(2), 182-189. https://doi.org/10.1038/nn996

Mudrik, L., Lamy, D., \& Deouell, L. Y. (2010). ERP evidence for context congruity effects during simultaneous object-scene processing. Neuropsychologia, 48(2), 507-517. https://doi.org/10.1016/j.neuropsychologia.2009.10.011

Mudrik, L., Shalgi, S., Lamy, D., \& Deouell, L. Y. (2014). Synchronous contextual irregularities affect early scene processing: Replication and extension. Neuropsychologia, 56, 447-458. https://doi.org/10.1016/j.neuropsychologia.2014.02.020

Niefind, F., \& Dimigen, O. (2016). Dissociating parafoveal preview benefit and parafovea-on-fovea effects during reading: A combined eye tracking and EEG study. Psychophysiology, 53(12), 1784-1798. https://doi.org/10.1111/psyp.12765

Nikolaev, A. R., Meghanathan, R. N., \& van Leeuwen, C. (2016). Combining EEG and eye movement recording in free viewing: Pitfalls and possibilities. Brain and Cognition, 107, 55-83. https://doi.org/10.1016/j.bandc.2016.06.004

Nuthmann, A. (2013). On the visual span during object search in real-world scenes. Visual Cognition, 21(7), 803-837. https://doi.org/10.1080/13506285.2013.832449

Nuthmann, A. (2014). How do the regions of the visual field contribute to object search in real-world scenes? Evidence from eye movements. Journal of Experimental Psychology: Human Perception and Performance, 40(1), 342-360. https://doi.org/10.1037/a0033854

Nuthmann, A., \& Einhäuser, W. (2015). A new approach to modeling the influence of image features on fixation selection in scenes. Annals of the New York Academy of Sciences, 1339(1), 82-96. https://doi.org/10.1111/nyas.12705

Nuthmann, A., \& Henderson, J. M. (2010). Object-based attentional selection in scene viewing. Journal of Vision, 10(8), :20, 1-19. https://doi.org/10.1167/10.8.20

Plöchl, M., Ossandón, J. P., \& König, P. (2012). Combining EEG and eye tracking: identification, characterization, and correction of eye movement artifacts in electroencephalographic data. Frontiers in Human Neuroscience, 6 , 1-23. https://doi.org/10.3389/fnhum.2012.00278

Rämä, P., \& Baccino, T. (2010). Eye fixation-related potentials (EFRPs) during object identification. Visual Neuroscience, 27(5-6), 187-192. https://doi.org/10.1017/S0952523810000283

Rayner, K. (2014). The gaze-contingent moving window in reading: Development and review. Visual Cognition, 22(3), 242-258. https://doi.org/10.1080/13506285.2013.879084

Satterthwaite, F. E. (1946). An Approximate Distribution of Estimates of Variance Components. Biometrics Bulletin, 2(6), 110-114. https://doi.org/10.1002/9780470057339.vai016

Serences, J. T. (2004). A comparison of methods for characterizing the event-related BOLD timeseries in rapid fMRI. NeuroImage, 21(4), 1690-1700. https://doi.org/10.1016/j.neuroimage.2003.12.021

Smith, N. J., \& Kutas, M. (2015a). Regression-based estimation of ERP waveforms: I. The rERP framework. Psychophysiology, 52(2), 157-168. https://doi.org/10.1111/psyp.12317

Smith, N. J., \& Kutas, M. (2015b). Regression-based estimation of ERP waveforms: II. Nonlinear effects, overlap correction, and practical considerations. Psychophysiology, 52(2), 169-181. https://doi.org/10.1111/psyp.12320

Smith, S. M., \& Nichols, T. E. (2009). Threshold-free cluster enhancement: Addressing problems of smoothing, threshold dependence and localisation in cluster inference. NeuroImage , 44(1), 83-98. https://doi.org/10.1016/j.neuroimage.2008.03.061

Stoll, J., Thrun, M., Nuthmann, A., \& Einhäuser, W. (2015). Overt attention in natural scenes: Objects dominate 
features. Vision Research, 107, 36-48. https://doi.org/10.1016/j.visres.2014.11.006

Thickbroom, G. W., Knezevič, W., Carroll, W. M., \& Mastaglia, F. L. (1991). Saccade onset and offset lambda waves: relation to pattern movement visually evoked potentials. Brain Research, 551(1-2), 150-156. https://doi.org/10.1016/0006-8993(91)90927-N

Underwood, G., \& Foulsham, T. (2006). Visual saliency and semantic incongruency influence eye movements when inspecting pictures. Quarterly Journal of Experimental Psychology (2006), 59(11), 1931-1949. https://doi.org/10.1080/17470210500416342

Underwood, G., Templeman, E., Lamming, L., \& Foulsham, T. (2008). Is attention necessary for object identification? Evidence from eye movements during the inspection of real-world scenes. Consciousness and Cognition, 17(1), 159-170. https://doi.org/10.1016/j.concog.2006.11.008

Viola, F. C., Debener, S., Thorne, J., \& Schneider, T. R. (2010). Using ICA for the analysis of multi-channel EEG data. In Simultaneous EEG and fMRI: Recording, Analysis, and Application: Recording, Analysis, and Application (pp. 121-133).

Võ, M. L.-H., \& Henderson, J. M. (2009). Does gravity matter? Effects of semantic and syntactic inconsistencies on the allocation of attention during scene perception. Journal of Vision, 9(3), :24, 1-15. https://doi.org/10.1167/9.3.24

Võ, M. L.-H., \& Henderson, J. M. (2011). Object-scene inconsistencies do not capture gaze: evidence from the flashpreview moving-window paradigm. Attention, Perception \& Psychophysics, 73(6), 1742-1753. https://doi.org/10.3758/s13414-011-0150-6

Võ, M. L.-H., \& Wolfe, J. M. (2013). Differential electrophysiological signatures of semantic and syntactic scene processing. Psychological Science, 24(9), 1816-1823. https://doi.org/10.1177/0956797613476955

Weidner, R., \& Müller, H. J. (2013). Dimensional weighting in cross-dimensional singleton conjunction search. Journal of Vision, 13(3), :25, 1-23. https://doi.org/10.1167/13.3.25

Weiss, B., Knakker, B., \& Vidnyánszky, Z. (2016). Visual processing during natural reading. Scientific Reports, 6, 26902. https://doi.org/10.1038/srep26902

Winkler, I., Debener, S., Müller, K.-R., \& Tangermann, M. (2015). On the influence of high-pass filtering on ICAbased artifact reduction in EEG-ERP. Proceedings of the Annual International Conference of the IEEE Engineering in Medicine and Biology Society, EMBS, 2015-Novem, 4101-4105. https://doi.org/10.1109/EMBC.2015.7319296

Wolfe, J. M., Alvarez, G. A., Rosenholtz, R., Kuzmova, Y. I., \& Sherman, A. M. (2011). Visual search for arbitrary objects in real scenes. Attention, Perception, and Psychophysics, 73(6), 1650-1671. https://doi.org/10.3758/s13414-011-0153-3

Wu, C. C., Wick, F. A., \& Pomplun, M. (2014). Guidance of visual attention by semantic information in real-world scenes. Frontiers in Psychology. https://doi.org/10.3389/fpsyg.2014.00054 


\section{Appendix}

Figure A1
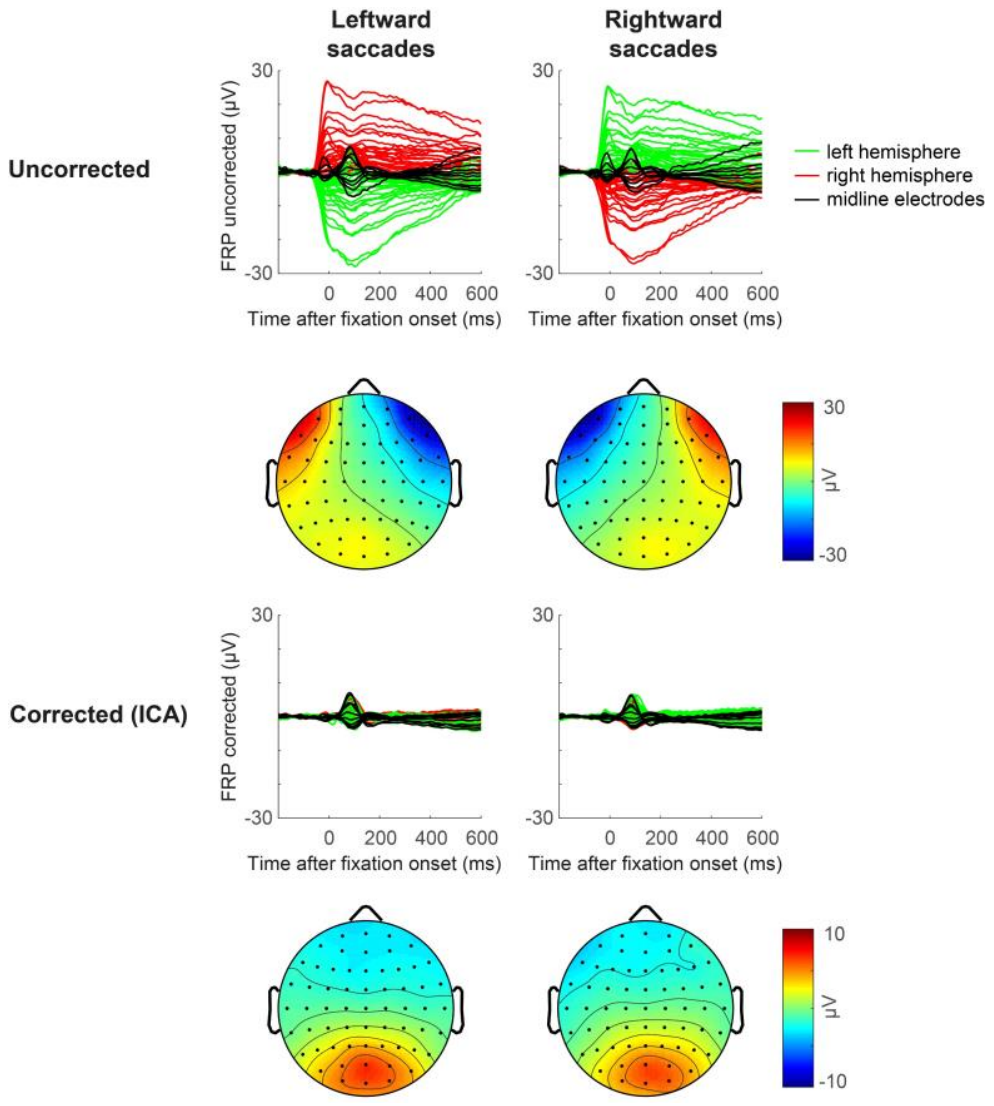

Figure A1. Results of ICA-based ocular artefact correction. Top panels show the FRP before ocular correction, separately for fixations following leftward and rightward saccades during scene viewing. Electrodes located over the left and right cerebral hemisphere are plotted in red and green, respectively, and electrodes on the sagittal midline are plotted in black. Scalp maps show the average voltages in a $20 \mathrm{~ms}$ window (72 to $92 \mathrm{~ms}$ ) centred on the peak of the fixation-related occipital lambda response (the P1 equivalent in FRPs). Lower panels show the same FRPs after ocular correction with ICA. Corneoretinal artefacts were almost fully removed by the ICA procedure meaning and the lambda response now becomes clearly visible in the scalp maps. 
Figure A2

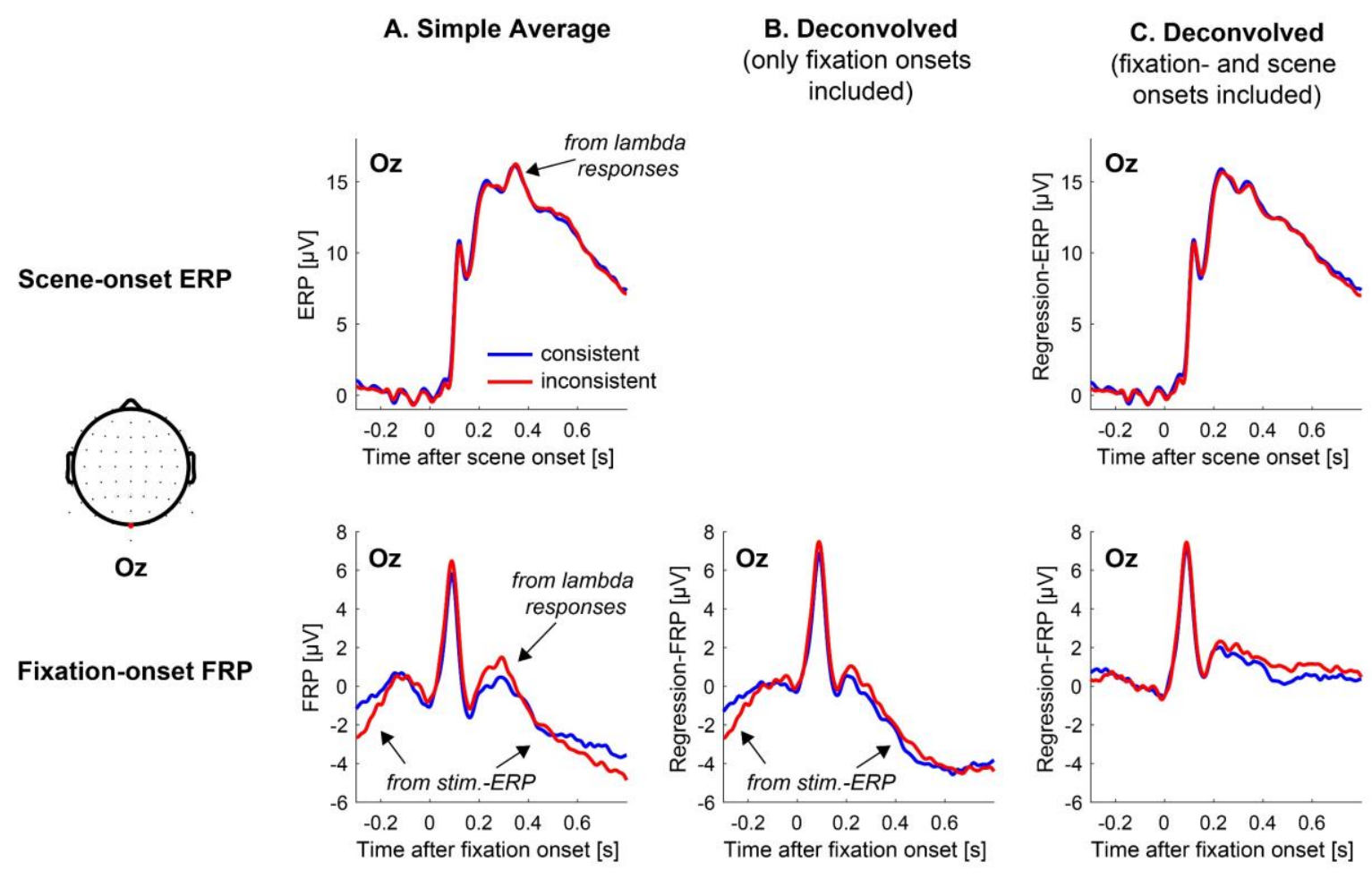

Figure A2. Controlling overlapping potentials and oculomotor covariates with linear deconvolution modelling in the unfold toolbox (Ehinger \& Dimigen, 2018). Shown here are both the ERP elicited by scene onset (upper row of panels) and the FRP elicited by the pre-target fixation $t-1$ (lower row of panels) at occipital electrode Oz. (A) The left column shows the result of a traditional ERP/FRP averaging; neither overlapping potentials nor saccade amplitude are controlled in these simple averages. The scene onset-ERP therefore contains overlapping activity (in particular the fixation-related P1, or lambda response) generated from the first fixation on the scene (most visible around $400 \mathrm{~ms}$, see arrow). Similarly, the baseline interval for the FRP curves is not flat, but strongly distorted by the stimulus-ERP that overlaps due to the preceding scene onset. Because fixations on inconsistent objects occurred on average earlier than those on consistent objects, it is important to control this overlap with the scene-ERP. (B) Same data, analysed with a linear deconvolution model in which only the overlapping potentials from neighbouring fixations were removed (see formula B below). Incoming saccade amplitude was also included as a linear covariate in the model, but the influence of this predictor was small due to the fact that saccade size was similar in both experimental conditions (Table 3). Because stimulus onset events were not included in this model, the distortions of the FRP baseline remain. (C) Same data, but now with both stimulus onset and fixation onsets included as events in the model (see formula $\mathrm{C}$ below). Overlapping potentials from scene onset are now also removed from the FRP, leaving the baseline interval essentially flat. Conversely, the distorting effects of overlapping FRPs (i.e. lambda responses) are removed from the stimulus-ERP (upper right plot). The model formulas (in Wilkinson notation) for the models depicted in the three columns above were:
A. Simple ERP and FRP average
B. Fixation events: $\quad \mathrm{FRP}=1+$ Congruency * Type + SaccadeAmp
Stimulus events: not modelled
C. Fixation events: $\quad$ FRP $=1+$ Congruency * Type + SaccadeAmp
Stimulus events: $\quad$ ERP $=1+$ Congruency 
Figure A3

A. Simple Average
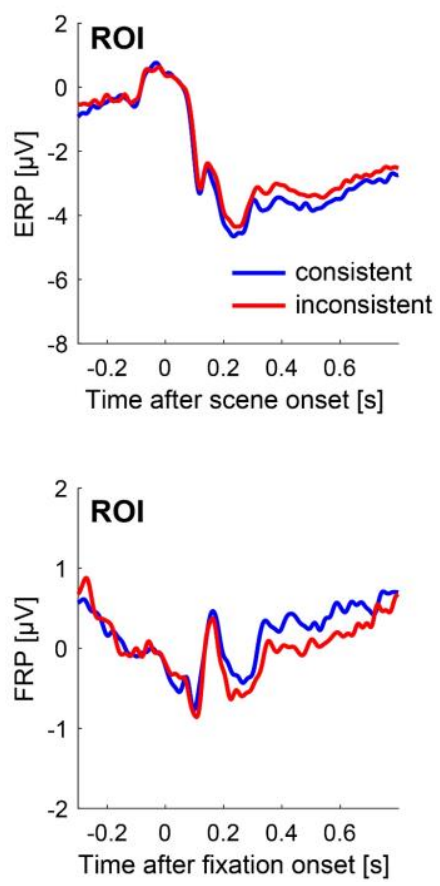

B. Deconvolved

(only fixation onsets included)
C. Deconvolved

(fixation- and scene onsets included)
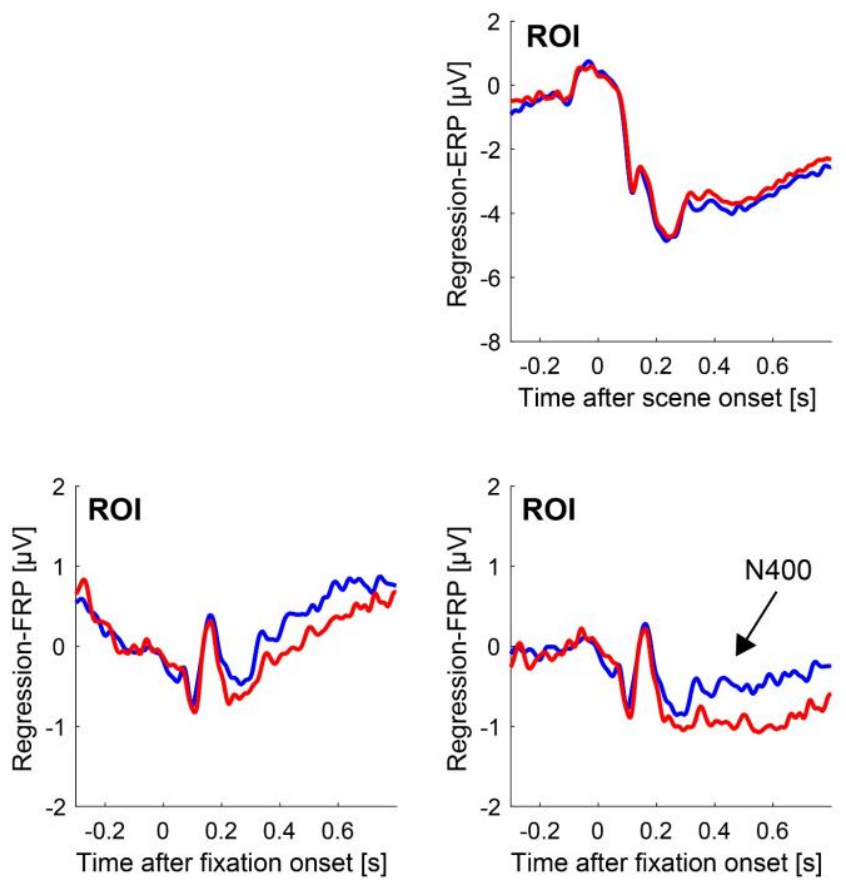

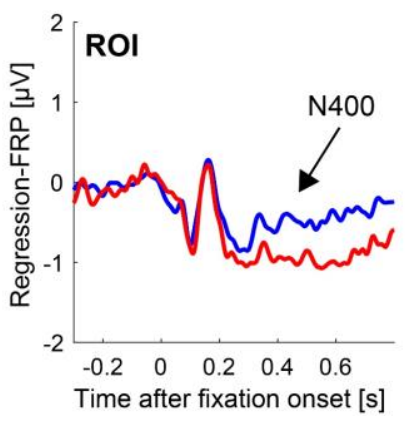

Figure A3. Same plots as Figure A2, but for the central ROI at which the object-scene consistency effects were strongest. Note that the N400 effect for consistent versus inconsistent objects on the pre-target fixation $(t-1)$ can be seen even in the raw averaged FRP waveforms (lower left panel) without any statistical control of overlapping potentials and saccade amplitude. Nevertheless, waveforms become much cleaner after the removal of overlapping activity from neighbouring fixations (middle column) and the scene onset (right column). 
Figure A4
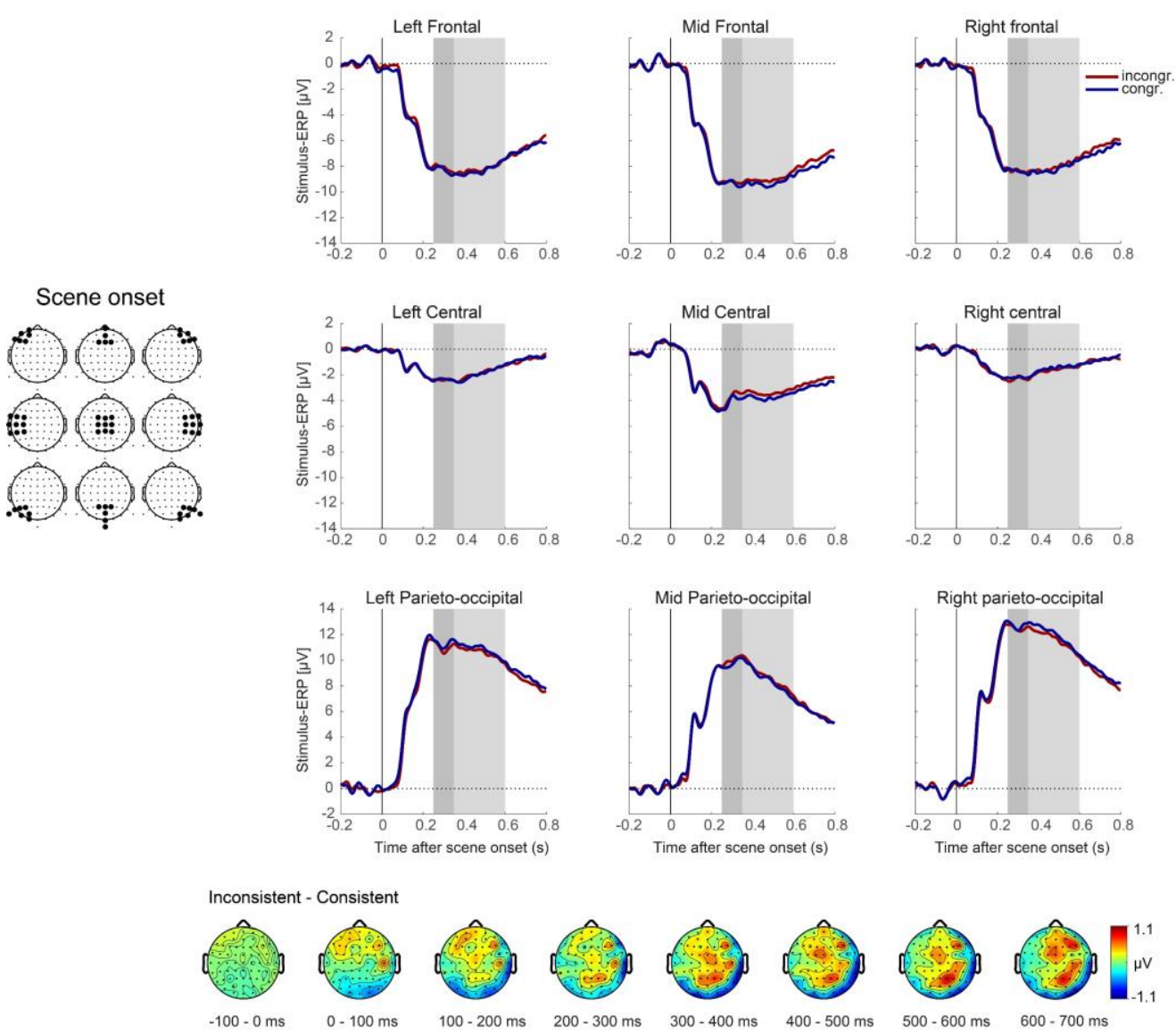

$100-200 \mathrm{~ms} \quad 200-300 \mathrm{~ms}$

$300-400 \mathrm{~ms}$

$500-600 \mathrm{~ms}$

$600-700 \mathrm{~ms}$

Figure A4. Grand-average scene-onset ERPs, aligned to the initial presentation of the scene stimulus. Waveforms are plotted separately for scenes containing a semantically consistent versus inconsistent target object. Electrodes are aggregated into nine regions-of-interest (ROIs, visualized on the left) that were adopted from previous research on object-scene consistency. Topographical maps show the scalp distribution of the difference (inconsistent minus consistent) in successive $100 \mathrm{~ms}$ time windows. 
Figure A5
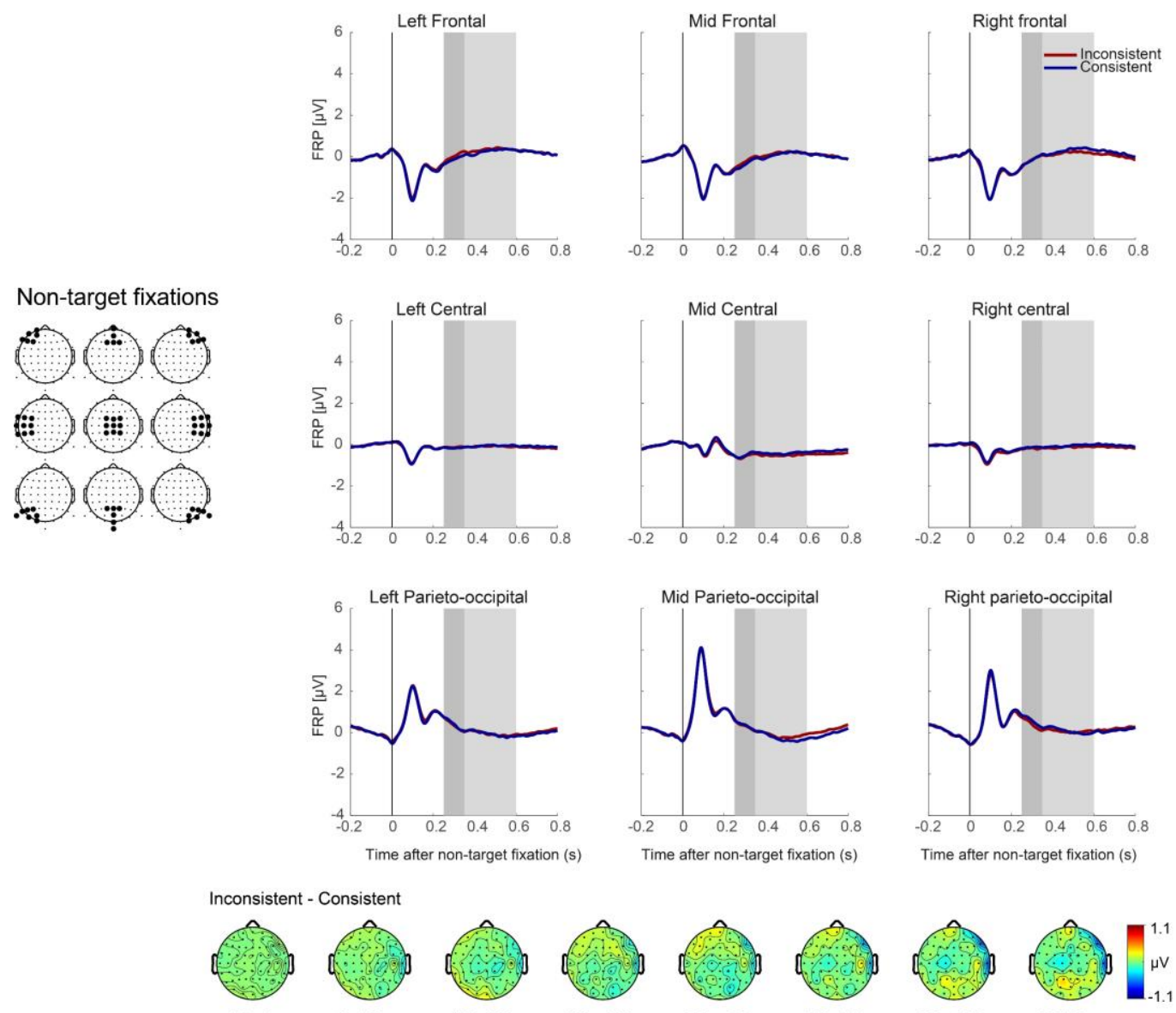

$-100-0 \mathrm{~ms}$

$0-100 \mathrm{~ms}$

$100-200 \mathrm{~ms} \quad 200-300 \mathrm{~ms}$

$300-400 \mathrm{~ms}$

$400-500 \mathrm{~ms}$

$500-600 \mathrm{~ms}$

$600-700 \mathrm{~ms}$

Figure A5. Grand-average FRPs aligned to the onset of non-target (nt) fixations on the scene. See caption of Figure A4 for further details. 
Figure A6
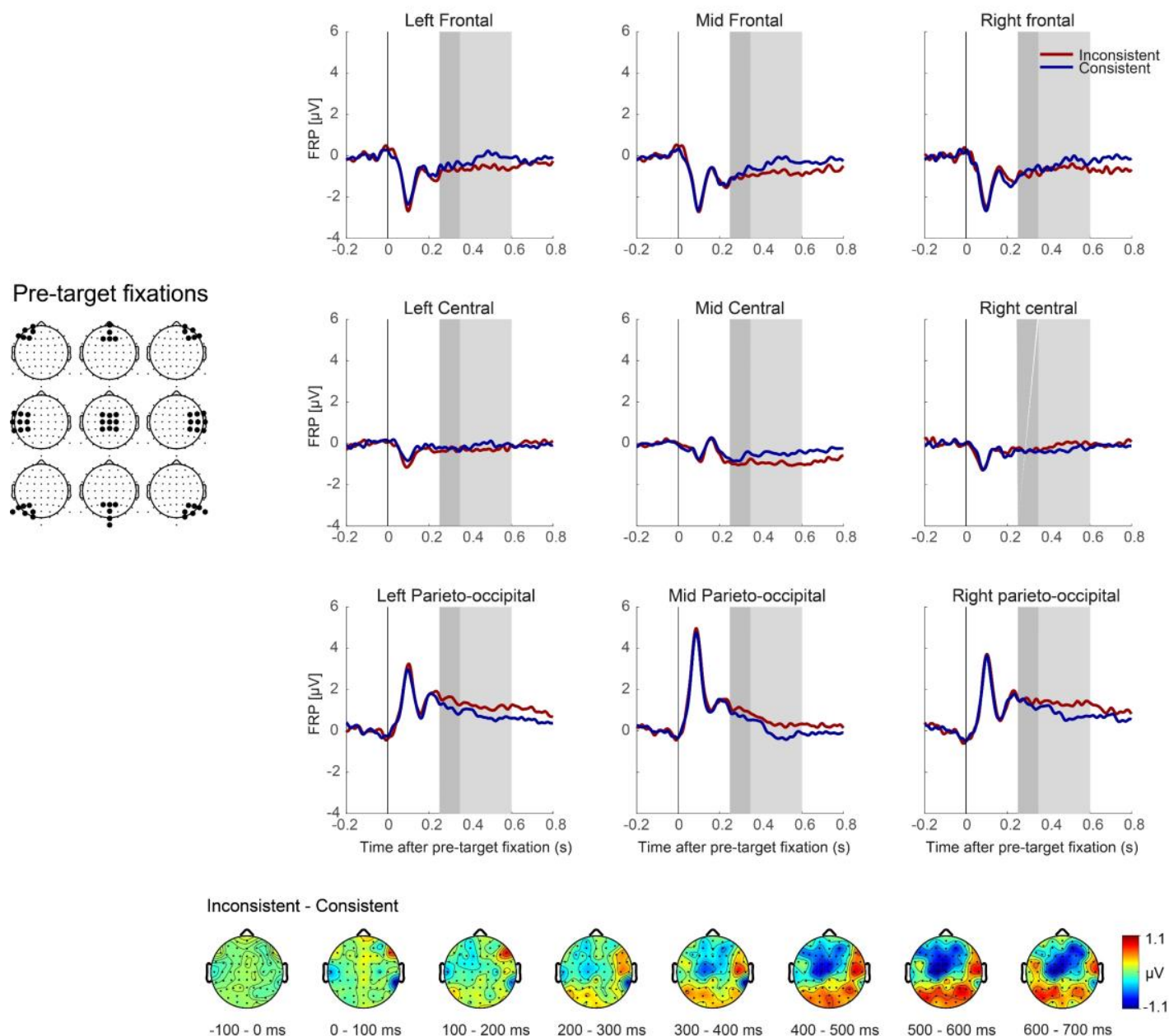

Figure A6. Grand-average FRPs aligned to the pre-target ( $t-1)$ fixations. See caption of Figure A4 for further details. 
Figure A7
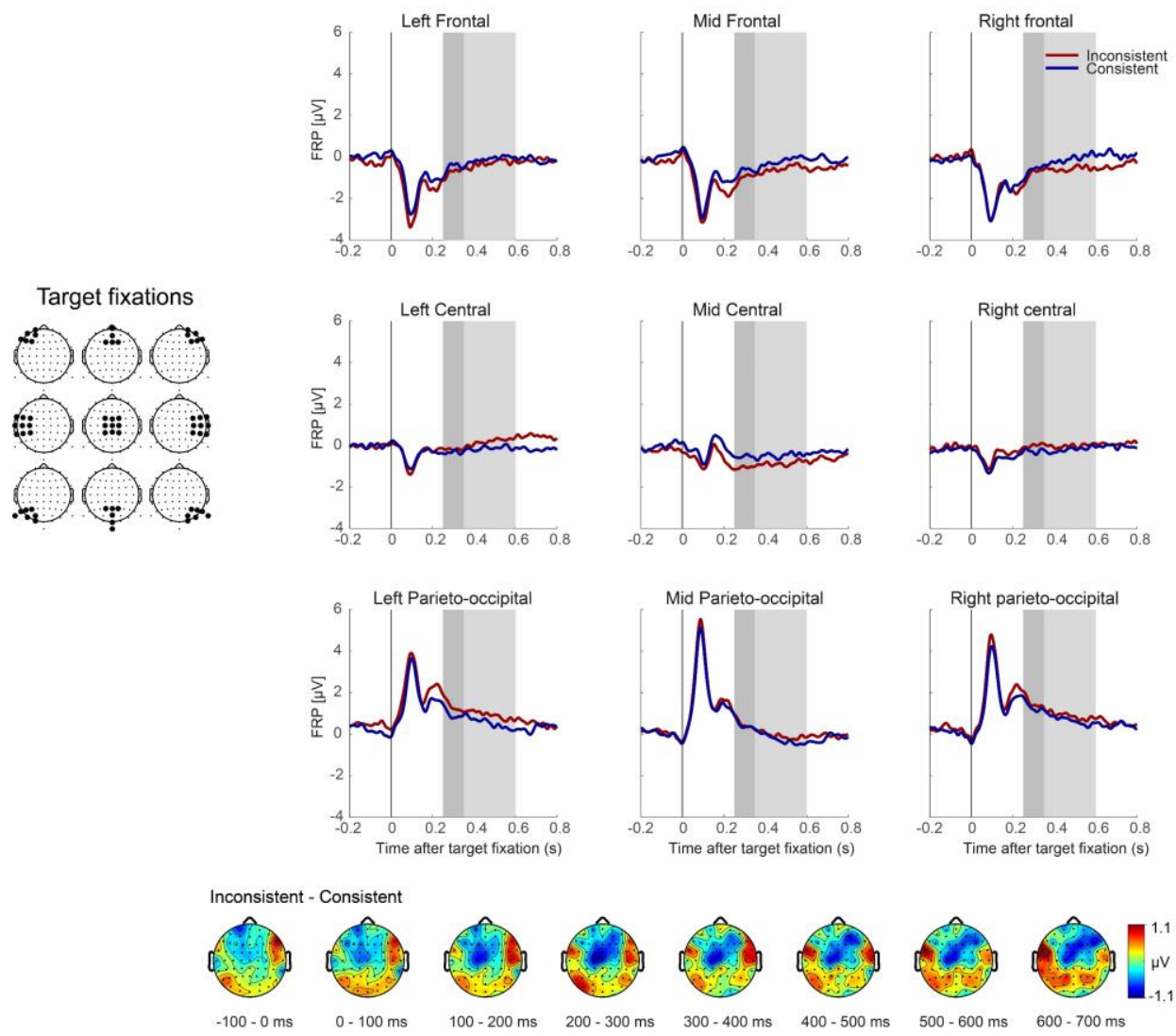

$-100-0 \mathrm{~ms}$

$300-400 \mathrm{~ms}$

400 - $500 \mathrm{~ms}$

$500-600 \mathrm{~ms}$

$600-700 \mathrm{~ms}$

Figure A7. Grand-average FRPs aligned to target $(t)$ fixations. See caption of Figure A4 for further details. 